\title{
Rho Kinase regulates neutrophil NET formation that is involved in UVB-induced skin inflammation
}

Minghui $\mathrm{Li}^{1,2,3 \dagger}$, Xing Lyu ${ }^{1,2,3 \dagger}$, James Liao ${ }^{4}$, Victoria P. Werth ${ }^{1,2}$, Ming-Lin Liu ${ }^{1,2}$

${ }^{1}$ Corporal Michael J. Crescenz VAMC, Philadelphia, PA, 19104, USA

${ }^{2}$ Department of Dermatology, Perelman School of Medicine, University of Pennsylvania,

Philadelphia, PA, 19104, USA

${ }^{3}$ Department of Rheumatology and Immunology, Tianjin Medical University General Hospital, Tianjin 300052, China

${ }^{4}$ Division of Cardiology, Department of Medicine, University of Chicago, Chicago, IL, USA

${ }^{\dagger}$ Contribution equally

Running title: Li et al. Rho kinase and NETosis

Key words: Rho kinase, Neutrophil, NETosis, UVB, Skin inflammation.

Correspondence to Ming-Lin Liu, MBBS, PhD, Department of Dermatology, Perelman School of Medicine, University of Pennsylvania, and Philadelphia Veterans Administration Medical center, Philadelphia, PA 19104, eMAIL: lium1@pennmedicine.upenn.edu, 


\begin{abstract}
Objective. Ultraviolet B (UVB) is an important trigger of skin inflammation and lupus with leukocyte recruitment to the inflamed skin. We recently reported the involvement of neutrophil NET formation in UVB-induced skin inflammation, and NETotic nuclear envelope rupture is driven by PKC $\alpha$-mediated nuclear lamin B disassembly. Actin cytoskeleton has been shown to be involved in NETosis with unclear mechanisms. Here, we investigated the role of Rho kinase (ROCK) and its downstream actomyosin cytoskeletal networks in PKC $\alpha$ nuclear translocation and NET formation, as well as the involvement of NETs in UVB-induced skin inflammation.
\end{abstract}

Methods. We studied the dynamic changes of ROCK and actomyosin cytoskeletal networks during NETosis induction and their role in $\mathrm{PKC} \alpha$ nuclear translocation. Using mice with hematopoietic specific ROCK1 deficiency, we investigated the effects of ROCK1 deficiency on NETosis, and its involvement in UVB-induced skin inflammation.

Results. We found the time course process of PKC $\alpha$ nuclear translocation was very well matched with the time course of actin polymerization and ROCK activation in early stage of NETosis induction. Importantly, inhibition of actin polymerization or key molecules of ROCK/MLCK/myosin pathway decreased nuclear translocation of $\mathrm{PKC} \alpha$ and NET formation. Genetic deficiency of ROCK1 inhibited neutrophil NETosis ex vivo and in vivo, decreased the extracellular display of NET-associated IL-17A, TNF $\alpha$, IFN $\gamma$, and IFN $\alpha$ in the inflamed skin, and ameliorated skin inflammation in UVB-irradiated mice with hematopoietic specific ROCK1 deficiency.

Conclusion. ROCK regulated NET formation in neutrophils through modulation of PKC $\alpha$ nuclear translocation via actomyosin cytoskeletal networks. ROCK1 deficiency ameliorated UVB-induced 
bioRxiv preprint doi: https://doi.org/10.1101/2021.05.16.444366; this version posted August 17, 2021. The copyright holder for this preprint

(which was not certified by peer review) is the author/funder, who has granted bioRxiv a license to display the preprint in perpetuity. It is made available under aCC-BY-NC-ND 4.0 International license.

skin inflammation by inhibition of NETosis and the extracellular display of NET-associated cytokines. 


\section{INTRODUCTION}

Ultraviolet B (UVB) is an important trigger of cutaneous and systemic lupus erythematosus (CLE, SLE) [1-4]. UVB overexposure causes photodamage of skin with recruitment of inflammatory cells, including neutrophils [5]. A recent study has indicated that neutrophils are the critical early infiltrating immune cells within cutaneous lupus lesion in mice [6]. Several studies with transcriptional profiling analysis of lupus patient samples also confirmed the importance of neutrophils [7], and a gradual enrichment of neutrophil transcripts during lupus disease progression [8]. Therefore, it is important to understand the role of neutrophils in UVB-induced skin inflammation. NETosis is a unique form of neutrophil cell death with release neutrophil extracellular traps (NETs) [9], which are involved in lupus pathogenesis $[10,11]$. However, the involvement of NETosis in UVB-induced skin inflammation and the relevant cellular mechanisms are not well understood.

Nuclear chromatin forms the backbone of NETs, thus nuclear envelope rupture is a required step for breaking the first physical barrier for nuclear chromatin release and extracellular NET formation $[9,11,12]$. We recently found that NETotic nuclear envelope rupture is driven by protein kinase $\mathrm{C}$ alpha (PKC $\alpha$ )-mediated phosphorylation and disassembly of nuclear lamin $\mathrm{B}$ [9] which is crucial to nuclear envelope integrity by forming highly organized meshworks surrounding the nuclear chromatin [13]. Furthermore, cyclin-dependent kinases (CDK)4/6 also control NETosis through regulation of lamin A/C phosphorylation [14]. Therefore, PKC $\alpha$ and CDK4/6 serve as NETotic lamin kinases $[9,12,14]$ for regulation of nuclear lamina disassembly, nuclear envelope rupture, and chromatin extracellular release. 
PKC $\alpha$ exists diffusely in the cytosol of the unstimulated cells, and the kinase can be translocated to the nucleus during activation [15]. Schmalz et al indicated that nuclear translocation of cytosolic $\mathrm{PKC} \alpha$ requires intact cytoskeleton [16]. As an upstream regulator of cytoskeleton, Rho kinase (ROCK) inhibition blocks nuclear translocation and activation of PKC [17]. Recently, we have shown that PKC $\alpha$ nuclear translocation is required for nuclear lamina disassembly and NET formation [9]. Publications demonstrated that functional cytoskeleton is required [18-21] for NETosis induction in the early stage of neutrophil stimulation, while actin cytoskeleton disassembly is required for plasma membrane rupture which is necessary for chromatin extracellular release [12, 22].

In the current study, we sought to study the dynamic changes of actin cytoskeleton and its upstream regulator ROCK, as well as their involvement in $\mathrm{PKC} \alpha$ nuclear translocation during NETosis induction. Furthermore, F-actin and myosin motors form actin-myosin (actomyosin) cytoskeletal networks that are involved in nucleocytoplasmic shuttling [23]. The actomyosin cytoskeletal networks can be regulated by activation of myosin II and myosin regulatory light chain kinase (MLCK) through the ROCK/MLCK/myosin pathway [24]. Therefore, we also investigated the involvement of ROCK/MLCK/myosin pathway in $\mathrm{PKC} \alpha$ nuclear translocation and NET formation in vitro. Importantly, we investigated the effects of ROCK1 deficiency on neutrophil NET formation in vivo and UVB-induced skin inflammation using mice with hematopoietic specific ROCK1 deficiency.

\section{METHODS}


Mice. ROCK1 deficient mice ROCK1 ${ }^{+/-}$on a C57BL/6J background were generated by Liao's laboratory as described before [25]. Since homozygous ( $\left.\mathrm{ROCK} 1^{-/-}\right)$mice always die perinatally, heterozygous mice were used in this study. C57BL/6J wildtype (WT) and CD45.1 (B6.SJL$\operatorname{Ptprc}^{a} P e p c^{b} /$ BoyJ) mice were purchased from Jackson Laboratory. All mice and their corresponding littermate controls were housed in a pathogen-free environment, and given food and water ad libitum. All animal experiments and animal protocols were approved by the Animal Care and Use Committee of University of Pennsylvania.

Generation of hematopoietic deficient mice by bone marrow transplantation (BMT). To generate mice with hematopoietic specific ROCK1 deficiency, or their corresponding background control mice, BMT was conducted according to our established BMT techniques by following the approved animal protocol, as described [26] with modification. In brief, the BM hematopoietic stem cells (HSCs, $>5 \times 10^{6}$ cells) were harvested from the femur and tibia of donor $\mathrm{ROCK} 1^{+/}$mice or their littermate WT (all 6-8-week-old, with CD45.2 congenic background). The donor HSCs $\left(>5 \times 10^{6}\right.$ cells) from either ROCK $1^{+/-}$or WT mice were transplanted into sex-matched CD45.1 recipient mice (all 6-8-week-old) which were pre-irradiated sub-lethally with two equal doses of 550 cGy (Total Body Irradiation 1100 cGy) at intervals of 18 hours [27, 28] with a Cs-137 Irradiator [26]. From one-week prior the BMT, mice were given medicated acidified water with antibiotics (sulfamethoxazole and trimethoprim) until 6 weeks post BMT. Mice were given soften food and kept in animal biosafety level 2 room immediately after irradiation and BMT for up to 6 weeks until UVB exposure. Peripheral blood chimerism was analyzed by flow cytometry analysis of reconstitution rate of CD45.2 cells in the peripheral blood of CD45.1 recipient mice at 4 weeks 
post BMT as a functional assay of HSC fitness. Only successfully transplanted mice (over $95 \%$ reconstitution) were used in later animal experiments.

UVB Exposure. The dorsal skins of CD45.1 mice with hematopoietic specific ROCK1 deficiency $\left(B M T-\mathrm{ROCK}^{+/-}\right)$, or their corresponding control mice (BMT-WT) were irradiated or not (sham groups) by UVB $\left(150 \mathrm{~mJ} / \mathrm{cm}^{2} /\right.$ day) for 5 consecutive days under anesthetization by peritoneal injected Ketamine/Xylazine [9]. Each group includes 6-8 mice with half-male and half-female studied. Animals were then sacrificed 24 hours after the last exposure. The whole dorsal skin samples, including epidermis, dermis, and subcutaneous fat were collected. Tissue sample sectioning was performed by the Penn Skin Biology and Diseases Resource-based Center. The skin sections from both UVB-irradiated and sham-irradiated mice were stained for hematoxylin and eosin $(H \& E)$. Infiltration of inflammatory cells was counted for the nucleated cells in the epidermis, dermis, or fat [29, 30].

Cell Culture and Treatment. Primary human neutrophils from peripheral blood or mouse bone marrow (BM) neutrophils from the femur and tibia BM of WT vs ROCK1 ${ }^{+/-}[25]$ were isolated as described in our publication [9]. Mouse BM mPMNs were isolated from the femur and tibia BM with a neutrophil isolation kit (Miltenyl). Human HL-60 cell line were differentiated into polymorphic nuclear granulocytes (dPMNs) in RPMI-1640 with 10\% FBS and 1.2\% DMSO for 5-6 days as described in our published study [9]. All of the above primary human or mouse neutrophils or dPMNs were treated with, either PMA at 50-100 nM, or physiological NETosis inducer platelet-activating factor (PAF) [9] at 5-10 $\mu \mathrm{M}$, for $1 \mathrm{~h}$ or $3 \mathrm{~h}$ without or with $30 \mathrm{~min}$ pretreatment with inhibitors of F-actin polymerization (Cytochalasin D), myosin II (Blebbistatin), 
myosin light chain (MLC) kinase (ML7), or ROCK (AS1892802, HA-1077, Y-27632), following by detection of NET formation or actin polymerization. Time course treatment of dPMNs by 50 nM PMA for 0 (or $5 \mathrm{~min}$ for adherence to plate for confocal microscopy analysis), 15, 30, 60, 120, and $180 \mathrm{~min}$, then cells were fixed with $2 \%$ paraformaldehyde or lysed with cell lysis buffer for different experiments. For detection of ROCK activity, the cell lysates from different time points were analyzed with ROCK activity detection kit (Millipore).

Assessment of NET Formation. All of the above primary human or mouse neutrophils or dPMNs were treated with either PMA $(50$ or $100 \mathrm{nM})$ or PAF $(5$ or $10 \mu \mathrm{M})$ for $3 \mathrm{~h}$. NET formation assessment and quantification was analyzed by either fluorometric NET quantification by SYTOX Green staining intensity, and/or immunofluorescent imaging quantification analysis by staining with impermeable DNA dye SYTOX Green for detection of NET formation, and the cellpermeable DNA dye SYTO Red to count the total number of neutrophils, as described before [9].

Flow cytometry analysis. For detection of polymerized filamentous actin (F-actin), the $2 \%$ paraformaldehyde (PFA) fixed neutrophils from different time points (time course) after PMA stimulation, or PMA-stimulated cells without or with pretreatment by ROCK inhibitors were stained by RFP-labeled phalloidin and analyzed with flow cytometry according to published method [31]. To detect the reconstitution rate of donor-derived cells in peripheral blood of BMT mice, blood samples were harvested from saphenous vein of all mice 4 weeks after BMT procedure. Then, erythrocytes were removed by hypotonic lysis $\left(0.2 \% \mathrm{NaCl}_{2}\right)$, and stained with PE-labeled anti-mouse CD45.1 and FITC-labeled anti-mouse CD45.2, following by flow cytometry analysis as described before [32]. 
Transmigration Assays. The pore transmigration ability of primary neutrophils from WT vs ROCK $1^{+/-}$mice was examined as described before [9] by using Boyden chamber, 24-well transwell plates with $3 \mu \mathrm{m}$ pore inserts, under stimulation with CXCL-1 (100 ng/ml). The pore transmigration ability was expressed as relative transmigration compared to that in neutrophils without treatment.

Fluorescent Immunocytochemistry and Immunohistochemistry analysis. For immunocytochemistry analysis, dPMNs or mouse BM neutrophils were treated without or with different inhibitors, then treated without or with PMA or PAF for 1-3h for study of PKC $\alpha$ nuclear translocation. Then the treated cells were stained by primary anti-total PKC $\alpha$ or anti-lamin B Abs, followed by secondary Abs with FITC or PE conjugation [9]. For the ex vivo NETosis induction with exhibition of NET-associated cytokines, the BM neutrophils from $\mathrm{ROCK} 1^{+/-}$vs WT were treated for $20 \mathrm{~h}$ without or with PAF, without or with isolated NETs (for IFN $\alpha$ induction). Then the treated cells were fixed with $2 \%$ PFA and permeabilized with $0.1 \%$ Triton X-100, and stained by primary antibodies against IL-17A, TNF $\alpha$, IFN $\gamma$, or IFN $\alpha$ with FITC- or Alexa488-conjugation (Biolegend or Bioss). The neutrophil marker Alexa-647-labeled anti-ly6G was used. DNA was stained with DAPI. The paraffin-embedded skin tissue sections were stained as described before [9]. The neutrophil markers Alexa-647-labeled anti-ly6G were used for detection of neutrophils and NETosis in combination with DNA staining with DAPI as described [9]. FITC- or Alexa488conjugated primary antibodies against IL-17A, TNF $\alpha$, IFN $\gamma$, or IFN $\alpha$ were used to stain cytokines (Biolegend or Bioss). Slides were mounted with Gold Antifade Mountant (Invitrogen). Confocal fluorescent images were analyzed with an Olympus Fluoview 1000 confocal microscope. 
Statistical analyses. GraphPad Prism 6 was used to perform statistical analysis. Normally distributed data are shown as means \pm standard deviations (SD). Comparisons between two groups were conducted with student $\mathrm{T}$ test. Comparisons amongst three or more groups were performed using ANOVA, followed by Student-Newman-Keuls (SNK) test. Pearson correlation analysis was used when applicable. Comparison between two groups was analyzed using Student's t-test. Statistical significance was considered at a level of $P$-value $<0.05$.

\section{RESULTS}

\section{Disruption of actin cytoskeleton or actomyosin cytoskeletal networks attenuated neutrophil NET formation.}

Nuclear envelope rupture is regulated by PKC $\alpha$-mediated lamin B disassembly [9, 12], and nuclear translocation of PKC $\alpha$ requires intact cytoskeleton $[16,21,33]$. In addition to actin cytoskeleton, actomyosin networks have been shown to be important in nucleocytoplasmic shuttling [23]. In the current study, we found that disruption of actin cytoskeleton filaments by inhibition of actin polymerization with cytochalasin $\mathrm{D}$, or disruption of actomyosin cytoskeletal networks by inhibition of myosin II or its upstream MLCK with Blebbistatin or ML7 correspondingly, significantly decreased PMA- or PAF-induced NETosis in human or mouse neutrophils (Figure 1A-C). Importantly, disruption of actin cytoskeleton or inhibition of myosin II or MLCK attenuated nuclear translocation of PKC $\alpha$ observed at 60 min after PMA stimulation (Fig 1D). Interestingly, the nuclei still remained multiple lobes with cytoplasmic retention of PKC $\alpha$ in these cells (Fig 1D). In contrast, we saw the swelling nuclei with disintegrated nuclear envelope in cells 
treated with PMA alone (Fig 1D). These results demonstrated that not only actin cytoskeleton, but also the actomyosin cytoskeletal networks, are important for nuclear translocation of PKC $\alpha$ and nuclear envelope rupture (Fig 1E), which is required for nuclear chromatin extrusion and NET formation $[9,12]$. In line, other studies also reported the involvement of actin cytoskeleton [34] or myosin II [35] in nuclear translocation of cytosolic molecules, and in actomyosin-dependent nucleocytoplasmic shuttling [23].

\section{ROCK regulated PKCa nuclear translocation by modulation of actin cytoskeleton polymerization in neutrophils.}

To understand the role of actin cytoskeleton and its upstream regulator ROCK in PKC $\alpha$ nuclear translocation, we studied the time course of PKC $\alpha$ nuclear translocation, and the dynamic changes of actin polymerization and Rho kinase activation in PMA-stimulated cells from 0 to $3 \mathrm{~h}$. We found that the diffusely distributed cytoplasmic $\mathrm{PKC} \alpha$ gradually translocated to the nucleus within 60 minutes after PMA stimulation (Fig 2A, image taken started at 5 min time point to allow neutrophils to adhere on plates). Alone with the nuclear accumulation of lamin kinase PKC $\alpha$ [9, 12], $\mathrm{PKC} \alpha$-mediated nuclear lamin B disassembly resulted in nuclear envelop disintegration at 60 min (Fig 2A), following by nuclear DNA extracellular extrusion from small rupture sites at 120 min (Fig 2A), and the extracellular release of large amount of nuclear DNA from the enlarged rupture of nuclear envelope at 180 min time point after PMA stimulation, resulting in NET formation with the collapse of the nuclear and plasma membrane $[9,12]$.

In parallel to the above changes in $\mathrm{PKC} \alpha$, we found a time-dependent increase of actin polymerization and F-actin filament formation at 15 and $30 \mathrm{~min}$, reached a peak by 60 min time 
point, following by gradual actin depolymerization at 120 and $180 \mathrm{~min}$ (Fig 2B). In line, a previous study reported that PMA can induce actin polymerization in neutrophils [33]. Importantly, the parallel changes of PKC $\alpha$ nuclear translocation and actin polymerization within 60 min after PMA stimulation (Fig 2A,B) support the role of actin cytoskeleton in $\mathrm{PKC} \alpha$ nuclear translocation in a previous study [16]. In addition, PMA stimulation increased ROCK activity at 15 and 30 min, and peaked at 60 min time point (Fig 2C), which are tightly matched with the dynamic changes of Factin polymerization within 60 min after PMA stimulation (Fig 2B). Interestingly, ROCK activity was gradually reduced, with significant decrease at $180 \mathrm{~min}$ time point as compared to those at 60 min (Fig 2C), corresponding to the gradual disassembly of actin cytoskeleton at 2-3h time points (Fig 2B). Therefore, the dynamic changes of actin polymerization/depolymerization (Fig 2B) were very well matched with the dynamic changes of ROCK activativity (Fig 2C) during the process of NETosis induction in PMA stimulated neutrophils, indicating the role of ROCK in actin cytoskeleton regulation. Furthermore, inhibition of ROCK activation by ROCK inhibitors (Y27632, HA1077, AS1892802) not only alleviated PMA-induced actin polymerization (Fig 2D), but also attenuated PKC $\alpha$ nuclear translocation, and inhibited nuclear envelope disintegration (Fig 2E). Therefore, PKC $\alpha$ nuclear translocation is mediated by actin cytoskeleton [16], and its upstream ROCK/MLCK/ myosin pathway (Fig 1E,2E,2F). In line, Hippenstiel demonstrated that inhibition of ROCK blocks nuclear translocation and activation of PKC in endothelial cells [17], and Matoba reported the role of ROCK in nuclear translocation of cytoplasmic molecules through regulation of actin cytoskeleton organization [36].

All in all, our results demonstrated that PMA stimulation induced actin cytoskeleton assembly in early stage (within $60 \mathrm{~min}$ ) of NETosis induction, during which actin cytoskeleton and actomyosin 
networks were involved in nuclear translocation of lamin kinase PKC $\alpha$ [16] which drives nuclear lamina disassembly for extrusion of nuclear DNA [9]. In line with the current study, several previous works also reported the involvement of actin cytoskeleton in NETosis [18-21]. In contrast to the early stage changes, the decreased ROCK activity at 180 min time points (Fig 2C) may result in the corresponding actin cytoskeleton disassembly (Fig 2B) and the consequent plasma membrane rupture for DNA extracellular release (Fig 2A) [22], as cortical cytoskeletal networks are important for plasma membrane integrity [12].

\section{Inhibition of ROCK by pharmacological inhibition or genetic deficiency attenuated NET formation in neutrophils in vitro or ex vivo.}

To explore the role of ROCK in NETosis, we found that inhibition of ROCK with different chemical inhibitors (Y27632, HA1077, AS1892802) significantly decreased NET formation in vitro in dPMNs (Fig 3A) and primary human neutrophils (Fig 3B) stimulated by PMA, or in mouse neutrophils stimulated by PAF (Fig 3C) detected by different approaches. Most importantly, genetic deficiency of ROCK1 inhibited NETosis ex vivo in BM neutrophils from ROCK1 ${ }^{+/-}$mice as compared to those from their WT littermates (Fig 3D,E). Taken together, increases of ROCK activation (Fig 2C) regulated actin polymerization (Fig 2B) which mediated PKC $\alpha$ nuclear translocation (Fig 2A) in a time-dependent manner within 60 min of PMA stimulation. Importantly, inhibition of ROCK attenuated actin polymerization (Fig 2D), decreased PKC nuclear accumulation (Fig 2E), and reduced NETosis in neutrophils in vitro or ex vivo (Fig 3A-E). Therefore, these results from in vitro and ex vivo experiments indicate that ROCK signal pathway regulated NETosis by modulation of nuclear translocation of lamin kinase PKC $\alpha$ and nuclear envelope rupture through regulation of the actomyosin cytoskeletal networks. 


\section{Hematopoietic specific ROCK1 deficiency protected mice from UVB-induced skin inflammation.}

To study the effects of ROCK1 on neutrophil NETosis in vivo and UVB-induced skin inflammation, we generated mice with hematopoietic specific ROCK1 deficiency by transplantation of BM HSCs from $\mathrm{ROCK} 1^{+/-}$mice or their WT littermates to CD45.1 mice, following by irradiation of these mice without or with UVB (Suppl Fig 1A). Four-weeks after the BMT procedure, reconstitution status of donor-derived cells (CD45.2) in peripheral blood of recipient CD45.1 mice was examined by flow cytometry for each BMT mouse. Only the recipient mice with over 95\% reconstitution rates (Suppl Fig 1B) were included for the following studies. The IHC staining of the infiltrated donor-derived neutrophils (CD45.2) and NET formation further confirmed the successful BMT with high reconstitution in skin lesions of UVB-irradiated CD45.1 recipient mice who received BMT of HSCs from WT donors (Suppl Fig 1C).

Interestingly, we found the decreased skin thickness of both epidermis and dermis, and reduced inflammatory cell infiltration in skin of UVB-irradiated BMT-ROCK $1^{+/-}$mice as compared to those in BMT-WT control mice (Fig 4A-D). Inflammatory cell infiltration in skin was positively correlated with dermal skin thickness in all experimental mice (Fig 4E). Importantly, IHC fluorescent staining of skin sections of these mice demonstrated the decreased NET formation in vivo in skin lesions of UVB-irradiated BMT-ROCK1 ${ }^{+/-}$mice as compared to those of BMT-WT control mice (Fig 4F,G). Interestingly, distribution of neutrophil NETs in skin was positively correlated with dermal skin thickness (Fig 4H), indicating the involvement of neutrophil NETs in skin inflammation. 
To understand the role of ROCK1 deficiency in skin inflammation and neutrophil NET formation, we found the significantly reduced infiltration of all inflammatory cells (Fig 4D) or neutrophils (Fig 4I) in the inflamed skin of UVB-irradiated BMT-ROCK $1^{+/-}$mice as compared to those in their BMT-WT controls. Wang et al reported that ROCK1 deletion reduced migration capability in bone marrow-derived macrophages from ROCK1 KO mice [37]. In the current study, we found the mildly, but significantly, decreased chemotactic migration ability in vitro in neutrophils with heterogeneous ROCK1 deficiency as compared to those from WT mice (Fig 4J). Cell migration is affected by cellular factors (including cytoskeleton) and environmental factors (including chemotactic signals) [38]. In the current study, the mildly reduced chemotactic migration ability in heterogeneous ROCK1 deficient neutrophils seems not enough to explain the ameliorated skin inflammation in UVB-irradiated BMT-ROCK1 $1^{+/-}$mice as compared to those in their BMT-WT controls (Fig 4A-D,J). Recent studies demonstrated that NETs can act as chemotactic factor to promote cancer metastasis [39] and NETs may enhance monocyte infiltration by induction of MCP1 expression in endothelial cells [40]. In the current study, we found the decreased NETosis in vitro in human or mouse neutrophils with ROCK inhibitors (Fig 3A-C), and ex vivo in neutrophils from ROCK $1^{+/-}$mice (Fig 3D,E). Most importantly, we found the significantly reduced percentage of NET formation in vivo among neutrophils infiltrated to the inflamed skin of UVBirradiated BMT-ROCK1+/- mice as compared to those in BMT-WT mice (Fig 4K). Therefore, the attenuated skin inflammation may, at least in part, be attributed to the reduced NET formation by ROCK1 deficiency (Fig 4A,F). Our published work has shown that neutrophil NETs can display IL-17A and TNF $\alpha$, cytokines with chemotactic properties, in UVB-induced skin inflammation [9]. 
Next, we sought to look the effects of ROCK1 deficiency on display of NET-associated cytokines in skin of UVB-irradiated BMT-ROCK1+/- mice.

\section{Hematopoietic-specific ROCK1 deficiency decreased the exhibition of pro-inflammatory cytokines by neutrophil NETs in BMT-ROCK1 ${ }^{+/-}$mice.}

To further study NET-related skin inflammation, we found that UVB exposure increased formation of NETs with exhibition of NET-associated IL-17A, TNF $\alpha$, IFN $\gamma$, or IFN $\alpha$ in the inflamed skin of BMT-WT mice (Fig 5A-I). Importantly, hematopoietic-specific ROCK1 deficiency can attenuate formation of neutrophil NETs in vivo and their display of above NET-associated cytokines in UVB-irradiated skin of BMT-ROCK1 ${ }^{+/}$mice (Fig 5A-I). Given that many cytokines have chemotactic properties for recruitment of inflammatory cells [41-43], here we found NETassociated IL-17A or TNF $\alpha$ in the inflamed skin in UVB-irradiated BMT-WT mice similar as we found in the UVB-irradiated WT mice in our recent publication [9]. Previous works have reported that IL-17A [41] together with TNFa [42] are known to synergistically induce leukocyte infiltration to the sites of inflammation [44]. Furthermore, NET-associated IFN $\gamma$ together with IL17A also promote leukocyte recruitment [43]. Thus, extracellular display of NET-associated IL17A, TNF $\alpha$, and IFN $\gamma$ in skin contributes to further recruitment of leukocytes and their accumulation in certain areas of the dermis, as well as propagation of inflammatory responses in the inflamed skin of UVB-irradiated BMT-WT mice (Fig 4F, 5C). However, there were fewer and diffusely distributed leukocytes in the skin of UVB-irradiated BMT-ROCK1 ${ }^{+/-}$mice (Fig 4F, 5C). Under UVB irradiation, the neutrophil NETs and their associated cytokines were accumulated with relatively high concentration in the extracellular matrix and likely contribute to further recruitment and accumulation of leukocytes in the skin of BMT-WT mice. In contrast, the non-netting 
neutrophils may secrete their cytokines that always quickly diffused to the blood stream and diluted by the circulation of UVB-irradiated BMT-ROCK $1^{+/-}$mice, and this feature may partly explain the fewer leukocyte infiltration in skin of these mice (Fig 4F, 5C). In addition to the above cytokines, we also detected NET-associated IFNa, an important cytokine that is crucial to pathogenesis of SLE [45, 46] and important to UVB-induced skin inflammation [30]. Interestingly, NET-associated IL-17A, TNF $\alpha$, IFN $\gamma$, and IFN $\alpha$ in skin were positively correlated with dermal skin thickness (Suppl Fig 2).

\section{ROCK1 genetic deficiency inhibited formation of NETs ex vivo and their exhibition of pro- inflammatory cytokines in neutrophils from ROCK1 deficient mice.}

Previous publications have reported that neutrophils can express and produce various cytokines, including IL-17A [43], TNF $\alpha$ [47], IFN $\gamma$ [48], or IFN $\alpha$ [49]. Studies from our and other groups have reported that neutrophil-expressed cytokines can be released with NETs [9, 49]. Recently, Agak et al demonstrated that extracellular traps released by TH17 cells can also be associated with IL-17 [50]. In the current study, we found the display of NET-associated cytokines in UVBirradiated skin (Fig 5). To determine if these NET-associated cytokines were derived from their parental neutrophils, we conducted ex vivo experiments by treatment of mouse neutrophils with PAF, a physiologic inducer of NETosis $[9,51]$. We found that NETs from PAF-stimulated WT neutrophils can display IL-17A, TNF $\alpha$, and IFN $\gamma$ to the extracellular space with their extracellular NET structures (Fig 6A,C,E). In contrast, ROCK1 deficiency attenuated NET formation with smaller display area of above cytokines expressed and enclosed by the non-netting neutrophils from $\mathrm{ROCK}^{+/-}$mice (Fig 6B,D,F). In addition, we also found IFN $\alpha$ expression in neutrophils and its extracellular display with NETs in WT neutrophils that treated with both PAF and isolated 
DNA NETs (NET+PAF), while ROCK1 deficiency reduced the display area of IFNa by the nonnetting neutrophils although they can express this cytokine when treated with NET+PAF. Under UVB irradiation, PAF released by keratinocytes can trigger neutrophils to form NETs that exhibit proinflammatory cytokines, which further recruited neutrophils, thus resulting in inflammatory cell accumulation in skin, particularly the epidermal-dermal junction area of the inflamed skin. In contrast, inhibition of NET formation by ROCK1 deficiency resulted in the intracellular retention of those cytokines, thus resulting in decreased and diffusely distributed leukocytes in the skin with ameliorated skin inflammation after UVB irradiation.

\section{DISCUSSION}

Photosensitive skin reaction is a complex inflammatory response to UVB-induced skin tissue damage [2, 4, 52, 53]. UVB overexposure causes epidermal keratinocyte apoptosis and DNA damage $[53,54]$. Accumulation of dead cell extracellular DNA may initiate autoimmune responses for lupus development [4]. However, some studies questioned about the role of UVB-induced keratinocyte apoptosis in lupus pathogenesis [55]. Beyond apoptosis, other type of cell death has also been shown to contribute to extracellular DNA accumulation, autoimmune responses, and lupus pathogenesis [56]. Histopathologic analysis of skin lupus indicates the potential interaction of between immune cell-epidermal keratinocytes [53, 57]. UVB irradiation of keratinocytes releases PAF, which contributes to the sustained inflammatory responses [58], i.e. inducing neutrophil recruitment and neutrophil NETosis in the inflamed skin [9]. Studies from our and other groups $[9,51]$ have shown that PAF can serve as a natural stimulus for NETosis induction in various conditions. On the other hand, studies from our and other groups have shown that UV 
exposure can induce neutrophil NET formation in vitro [9, 59]. Therefore, neutrophil NET formation in vivo in UVB-irradiated skin may be attributed to indirect effects of UVB by PAF [58] and direct effects of UVB irradiation $[9,59]$ on neutrophils in the inflamed skin.

Over the past decade, various signaling pathways have been reported to be involved in NETosis, i.e., NADPH oxidase (NOX)-dependent vs -independent, reactive oxygen species (ROS)dependent vs -independent etc [60-62]. Given that nuclear chromatin forms the backbone of NETs, the nucleus is the root of the nuclear DNA extracellular traps [12, 63-67]. Thus, decondensation of nuclear chromatin is required for its externalization, while rupture of the nuclear envelope and plasma membrane are the necessary steps for removal of the membrane barriers for nuclear DNA discharge and extracellular NET formation [12]. Therefore, these key cellular morphological changes, including nuclear chromatin, nuclear envelope, and plasma membrane, are required for extracellular DNA NET formation [9, 12, 14, 66, 67] no matter for NOX- or ROS-dependent or independent NETosis.

To understand the mechanisms for NETotic nuclear envelope rupture, we have recently reported that nuclear envelope rupture and chromatin extracellular release is driven by PKC $\alpha$-mediated nuclear lamin B disassembly [9]. In the current study, we found that nuclear translocation of cytosolic PKCa [9, 15] was regulated by actin cytoskeleton [16], actomyosin cytoskeletal networks and their upstream ROCK in the early stage of NETosis induction. Several previous studies have reported that NETosis induction requires functional cytoskeleton $[18,21]$, and genetic disruption of actin polymerization by a deficiency of Wiskott-Aldrich syndrome protein blocks NET formation [19]. In line with our findings, other studies also report the involvement of actin 
cytoskeleton filament [34], or myosin II [35], or actomyosin-dependent [23] nucleocytoplasmic shuttling. Matoba et al reported the role of ROCK in nuclear translocation of cytoplasmic molecules through regulation of actin cytoskeleton organization [36]. Therefore, the current study provides a potential cellular mechanistic explanation for the role of actin cytoskeleton in early stage of NETosis induction [18, 21], in which nuclear translocation of NETotic lamin kinase PKC $\alpha$ is mediated by actin cytoskeleton [16], and its upstream ROCK signaling pathway [17] during NETosis. The current work along with previous studies suggest that actomyosin cytoskeletal networks may serve as a "transportation system" for nuclear translocation of NETotic lamin kinase $\mathrm{PKC} \alpha[16]$, which phosphorylates and disassembles nuclear lamina, resulting in nuclear envelope rupture and extracellular release of nuclear DNA. In addition to their role in nuclear-cytoplasmic shuttling [23], ROCK and its-regulated actomyosin cytoskeletal networks may also enable nuclear deformation and contribute to nuclear envelope rupture by the forces generated by the cytoskeletal networks [68]. However, it is still unclear if actomyosin cytoskeletal networks also contribute to nuclear envelope rupture by tearing the disassembled nuclear lamina with their "forces".

Plasma membrane is the final barrier for extracellular release of nuclear chromatin and NET formation. The cell cortex is a layer of cytoskeletal networks underneath the plasma membrane, consisting of F-actin filaments, myosin motors, and actin-binding proteins [69]. The contractile forces generated by cortical cytoskeletal networks are important for the maintenance of plasma membrane integrity [69]. In contrast to ROCK activation and actin cytoskeleton polymerization in early stage of NETosis induction, their dynamic changes after the peak time point (60 min) turned to the gradually decrease in ROCK activity with actin cytoskeleton disassembly during the late 
stage NETosis induction in the current study. The late stage actin cytoskeleton disassembly may contribute to the plasma membrane rupture with NET release as reported in a recent study [22].

UVB-induced skin inflammation manifested as increased skin thickness, enhanced infiltration of inflammatory cells and elevated expression of proinflammatory cytokines. ROCK and its regulated actin cytoskeletal networks serve as cellular factors to modulate cell migration [38]. Wang and colleagues demonstrated that macrophages with ROCK1 deletion from ROCK1 ${ }^{-/}$mice showed significantly impaired chemotactic migration ability [37]. In the current study, the neutrophils with heterogeneous ROCK1 deficiency from $\mathrm{ROCK}^{+/-}$mice demonstrated mildly decreased transmigration ability ex vivo in transwell experiments. In vivo, the UVB irradiated ROCK $1^{+/-}$ mice showed the significantly reduced leukocyte infiltration with diffusely distributed non-netting neutrophils in skin, suggesting the reduced ROCK1 expression in leukocytes of ROCK1 ${ }^{+/-}$mice [25] can still partly regulate actomyosin cytoskeletal networks for driving cell movement. In a mean time, the reduced ROCK1 expression can inhibit NETosis in neutrophils through interfering cytoplasmic PKCa nuclear translocation and inducing nuclear envelope rupture [9].

Our published work has demonstrated the involvement of NETs and NET-associated cytokines in UVB-induced skin inflammation [9]. In the current study, ROCK1 deficiency can decrease NET formation and its display of NET-associated cytokines in vivo in skin of the UVB-irradiated mice. The decreased extracellular exhibition of NET-associated IL-17A, TNF $\alpha$, and IFN $\gamma$ may contribute to the reduced inflammatory cell infiltration and accumulation in the UVB-irradiated skin, as IL-17A vs TNF $\alpha$ are known to synergistically sustain neutrophil recruitment to the sites of inflammation [44], and IL-17A together with IFN $\gamma$ also promote neutrophil recruitment [43] to 
the inflamed skin. In contrast to the freely secreted cytokines that can diffuse to the circulation with large dilution with circulating blood, the NET-associated cytokines may retain in the local skin with a relatively high concentration for longer period of time, thus sustaining and propagating inflammatory responses in UVB-irradiated skin. Our recent publication demonstrated that blockage of TNF $\alpha$ with anti-TNF $\alpha$ significantly inhibited UVB-induced recruitment of inflammatory cells [70]. Using mice with genetic deficiency, Li et al reported that both IL-17A and IFN- $\gamma$ can be produced by neutrophils and may positively regulate neutrophil transmigration to the site of inflammation [43]. All of the above support the role of NET-associated IL-17A, TNF $\alpha$, or IFN $\gamma$ cytokines in recruitment of inflammatory cells to the inflamed skin of UVB irradiated BMT-WT mice, while inhibition of neutrophil NET formation with decreased extracellular display of NET-associated cytokines may at least partly explain the attenuated infiltration of leukocytes in the inflamed skin of BMT-ROCK1 $1^{+-}$mice. In line with the current study, recent publications demonstrated that NETs can act as chemotactic factor with NET-associated components, i.e. human LL-37 [71] or mouse CRAMP [72], to promote cell migration, including cancer metastasis [39]. In addition, NETs may enhance monocyte infiltration by induction of MCP1 expression in endothelial cells [40].

In addition to the above discussed chemotactic effects of NET-associated cytokines, NETassociated IFN $\gamma$ and IFN $\alpha$ not only contributes to UVB-induced skin inflammation [53, 73], but is also involved in lupus pathogenesis [74-76]. Although plasmacytoid dendritic cells are known to be professional cells for IFN $\alpha$ production, recent works indicate that neutrophils may also express IFN $\alpha$ in the presence of chromatin [49]. In the current study, we found NETotic neutrophils express IFN $\alpha$ in vivo in skin of UVB-irradiated BMT-WT mice, probably triggered by chromatin released 
from NETotic neutrophils nearby in the inflamed skin, while ROCK1 deficiency may decrease IFN $\alpha$ display through inhibition of NET formation in BMT-ROCK $1^{+/-}$mice. This is also confirmed by our ex vivo experiments in which IFNa expression in neutrophils can be produced by costimulation with PAF and isolated NETs. Since IFNa is a major driver of lupus pathogenesis, our results suggest that NETs and their associated IFN $\alpha$ may contribute to photosensitivity in lupus. Isgro et al reported the enhanced ROCK activation in SLE patients with increased Th17 cell differentiation, and inhibition of ROCK attenuates IL-17 production in SLE patients [77]. Thus, Rozo and colleagues indicated that ROCK might be a potential target for regulation of $\mathrm{T}$ cell dysfunction in SLE [78], while the current study demonstrated that ROCK might be a potential target for UVB-induced skin inflammation due to its role in regulation of NET formation.

Taken together, ROCK regulated NET formation in neutrophils by modulation of nuclear translocation of $\mathrm{PKC} \alpha$ through actomyosin cytoskeletal networks. ROCK1 deficiency ameliorates UVB-induced skin inflammation by attenuation of NET formation and its display of NETassociated cytokines. The current study demonstrates the contribution of NETosis to UVB-induced skin inflammation and photosensitivity in lupus, thus providing insights into novel therapeutics in NETosis-related human diseases, including lupus. 


\section{AUTHOR CONTRIBUTIONS}

Liu conceived and designed the study. Li, Lyu, Liu performed the laboratory experiments and acquired the experimental data. For the microscopic image analysis, Liu, Lyu, Li took the images, Lyu and Li conducted further quantification and statistical analysis. Liu, Lyu, and Werth analyzed and interpreted the data and finalized the paper. Li, Lyu, Liao, Werth and Liu were involved in preparation and revision of the manuscript. All authors read and approved the final manuscript.

\section{ACKNOWLEDGEMENTS}

The authors would like to acknowledge Penn Skin Biology and Diseases Resource-based Center for their skin histology service. The authors would thank Debra A. Pawlowski (animal facility, Philadelphia VA Medical Center) for her kindly help with our animal experiments. This work was supported by Lupus Research Alliance (416805) and NIH R21AI144838 (to MLL), the Veterans Affairs Merit Review Award (to VPW), and NIH HL052233 and HL136962 (to JL).

\section{CONFLICT OF INTEREST}

The authors have no conflict of interests to declare. 


\section{References}

1. Werth, V.P. et al. (2004) Photosensitivity in rheumatic diseases. J Investig Dermatol Symp Proc 9 (1), 57-63.

2. Doerner, J. et al. (2016) Fn14 deficiency protects lupus-prone mice from histological lupus erythematosus-like skin inflammation induced by ultraviolet light. Exp Dermatol 25 (12), 969-976.

3. Deng, G.M. and Tsokos, G.C. (2015) Pathogenesis and targeted treatment of skin injury in SLE. Nat Rev Rheumatol 11 (11), 663-9.

4. Wenzel, J. (2019) Cutaneous lupus erythematosus: new insights into pathogenesis and therapeutic strategies. Nat Rev Rheumatol 15 (9), 519-532.

5. Rodero, M.P. et al. (2014) Differential effects of ultraviolet irradiation in neonatal versus adult mice are not explained by defective macrophage or neutrophil infiltration. J Invest Dermatol 134 (7), 1991-7.

6. Han, X. et al. (2019) PD-1H (VISTA)-mediated suppression of autoimmunity in systemic and cutaneous lupus erythematosus. Sci Transl Med 11 (522).

7. Panousis, N.I. et al. (2019) Combined genetic and transcriptome analysis of patients with SLE: distinct, targetable signatures for susceptibility and severity. Ann Rheum Dis 78 (8), 10791089.

8. Banchereau, R. et al. (2016) Personalized Immunomonitoring Uncovers Molecular Networks that Stratify Lupus Patients. Cell 165 (3), 551-65.

9. Li, Y. et al. (2020) Nuclear envelope rupture and NET formation is driven by PKC $\alpha$-mediated lamin B disassembly. EMBO Rep 21 (e48779).

10. Kahlenberg, J.M. et al. (2013) Neutrophil extracellular trap-associated protein activation of the NLRP3 inflammasome is enhanced in lupus macrophages. J Immunol 190 (3), 1217-26.

11. Lood, C. et al. (2016) Neutrophil extracellular traps enriched in oxidized mitochondrial DNA are interferogenic and contribute to lupus-like disease. Nat Med 22 (2), 146-53.

12. Liu, M.L. et al. (2021) Recent progress in the mechanistic understanding of NET formation in neutrophils. FEBS J.

13. Goldberg, M.W. et al. (2008) Filaments made from A- and B-type lamins differ in structure and organization. J Cell Sci 121 (Pt 2), 215-25.

14. Amulic, B. et al. (2017) Cell-Cycle Proteins Control Production of Neutrophil Extracellular Traps. Dev Cell 43 (4), 449-462.

15. Zauli, G. et al. (1996) Nuclear translocation of protein kinase C-alpha and -zeta isoforms in HL-60 cells induced to differentiate along the granulocytic lineage by all-trans retinoic acid. Br J Haematol 93 (3), 542-50.

16. Schmalz, D. et al. (1996) Transport of protein kinase $C$ alpha into the nucleus requires intact cytoskeleton while the transport of a protein containing a canonical nuclear localization signal does not. J Cell Sci 109 ( Pt 9), 2401-6.

17. Hippenstiel, S. et al. (1998) Rho protein inhibition blocks protein kinase $\mathrm{C}$ translocation and activation. Biochem Biophys Res Commun 245 (3), 830-4.

18. Neeli, I. et al. (2009) Regulation of extracellular chromatin release from neutrophils. J Innate Immun 1 (3), 194-201.

19. Stojkov, D. et al. (2017) ROS and glutathionylation balance cytoskeletal dynamics in neutrophil extracellular trap formation. J Cell Biol 216 (12), 4073-4090. 
20. Neubert, E. et al. (2018) Chromatin swelling drives neutrophil extracellular trap release. Nat Commun 9 (1), 3767.

21. Liu, M.L. (2020) Functional actin cytoskeleton is required in early stage of NETosis induction. Proc Natl Acad Sci U S A 117 (37), 22653-22654.

22. Thiam, H.R. et al. (2020) NETosis proceeds by cytoskeleton and endomembrane disassembly and PAD4-mediated chromatin decondensation and nuclear envelope rupture. Proc Natl Acad Sci U S A 117 (13), 7326-7337.

23. Alisafaei, F. et al. (2019) Regulation of nuclear architecture, mechanics, and nucleocytoplasmic shuttling of epigenetic factors by cell geometric constraints. Proc Natl Acad Sci U S A 116 (27), 13200-13209.

24. Somlyo, A.P. and Somlyo, A.V. (2003) Ca2+ sensitivity of smooth muscle and nonmuscle myosin II: modulated by G proteins, kinases, and myosin phosphatase. Physiol Rev 83 (4), 1325-58.

25. Rikitake, Y. et al. (2005) Decreased perivascular fibrosis but not cardiac hypertrophy in ROCK1+/- haploinsufficient mice. Circulation 112 (19), 2959-65.

26. Duran-Struuck, R. and Dysko, R.C. (2009) Principles of bone marrow transplantation (BMT): providing optimal veterinary and husbandry care to irradiated mice in BMT studies. $\mathrm{J}$ Am Assoc Lab Anim Sci 48 (1), 11-22.

27. Down, J.D. et al. (1991) Syngeneic and allogeneic bone marrow engraftment after total body irradiation: dependence on dose, dose rate, and fractionation. Blood 77 (3), 661-9.

28. Marino, R. et al. (2013) Delayed marrow infusion in mice enhances hematopoietic and osteopoietic engraftment by facilitating transient expansion of the osteoblastic niche. Biol Blood Marrow Transplant 19 (11), 1566-73.

29. Menke, J. et al. (2008) Sunlight triggers cutaneous lupus through a CSF-1-dependent mechanism in MRL-Fas(lpr) mice. J Immunol 181 (10), 7367-79.

30. Sontheimer, C. et al. (2017) Ultraviolet B Irradiation Causes Stimulator of Interferon GenesDependent Production of Protective Type I Interferon in Mouse Skin by Recruited Inflammatory Monocytes. Arthritis Rheumatol 69 (4), 826-836.

31. Cano, M.L. et al. (1992) Mechanisms responsible for F-actin stabilization after lysis of polymorphonuclear leukocytes. J Cell Biol 116 (5), 1123-34.

32. Liu, M.L. et al. (2007) Cholesterol enrichment of human monocyte/macrophages induces surface exposure of phosphatidylserine and the release of biologically-active tissue factorpositive microvesicles. Arterioscler Thromb Vasc Biol 27 (2), 430-5.

33. Downey, G.P. et al. (1992) Phorbol ester-induced actin assembly in neutrophils: role of protein kinase C. J Cell Biol 116 (3), 695-706.

34. Yamamura, S. et al. (2020) Yes-associated protein 1 translocation through actin cytoskeleton organization in trophectoderm cells. Dev Biol 468 (1-2), 14-25.

35. Thomas, D.G. et al. (2015) Non-muscle myosin IIB is critical for nuclear translocation during 3D invasion. J Cell Biol 210 (4), 583-94.

36. Matoba, K. et al. (2014) Rho-kinase regulation of TNF-alpha-induced nuclear translocation of NF-kappaB RelA/p65 and M-CSF expression via p38 MAPK in mesangial cells. Am J Physiol Renal Physiol 307 (5), F571-80.

37. Wang, H.W. et al. (2008) Deficiency of ROCK1 in bone marrow-derived cells protects against atherosclerosis in LDLR-/- mice. FASEB J 22 (10), 3561-70.

38. Qu, F. et al. (2019) Cell migration: implications for repair and regeneration in joint disease. Nat Rev Rheumatol 15 (3), 167-179. 
39. Yang, L. et al. (2020) DNA of neutrophil extracellular traps promotes cancer metastasis via CCDC25. Nature 583 (7814), 133-138.

40. Hofbauer, T.M. et al. (2020) Neutrophil Extracellular Traps Induce MCP-1 at the Culprit Site in ST-Segment Elevation Myocardial Infarction. Front Cell Dev Biol 8, 564169.

41. Roussel, L. et al. (2010) IL-17 promotes p38 MAPK-dependent endothelial activation enhancing neutrophil recruitment to sites of inflammation. J Immunol 184 (8), 4531-7.

42. Smart, S.J. and Casale, T.B. (1994) Pulmonary epithelial cells facilitate TNF-alpha-induced neutrophil chemotaxis. A role for cytokine networking. J Immunol 152 (8), 4087-94.

43. Li, L. et al. (2010) IL-17 produced by neutrophils regulates IFN-gamma-mediated neutrophil migration in mouse kidney ischemia-reperfusion injury. J Clin Invest 120 (1), 331-42.

44. Griffin, G.K. et al. (2012) IL-17 and TNF-alpha sustain neutrophil recruitment during inflammation through synergistic effects on endothelial activation. J Immunol 188 (12), 628799.

45. Crow, M.K. (2014) Type I interferon in the pathogenesis of lupus. J Immunol 192 (12), 545968.

46. Crow, M.K. et al. (2018) Type I Interferons in Autoimmune Disease. Annu Rev Pathol.

47. Hilda, J.N. and Das, S.D. (2016) TLR stimulation of human neutrophils lead to increased release of MCP-1, MIP-1alpha, IL-1beta, IL-8 and TNF during tuberculosis. Hum Immunol 77 (1), 63-67.

48. Yamada, M. et al. (2011) Interferon-gamma production by neutrophils during bacterial pneumonia in mice. Am J Respir Crit Care Med 183 (10), 1391-401.

49. Lindau, D. et al. (2013) TLR9 independent interferon alpha production by neutrophils on NETosis in response to circulating chromatin, a key lupus autoantigen. Ann Rheum Dis.

50. Agak, G.W. et al. (2021) Extracellular traps released by antimicrobial TH17 cells contribute to host defense. J Clin Invest 131 (2).

51. Farley, K. et al. (2012) A serpinB1 regulatory mechanism is essential for restricting neutrophil extracellular trap generation. J Immunol 189 (9), 4574-81.

52. Shipman, W.D. et al. (2018) A protective Langerhans cell-keratinocyte axis that is dysfunctional in photosensitivity. Sci Transl Med 10 (454).

53. Wolf, S.J. et al. (2018) Human and Murine Evidence for Mechanisms Driving Autoimmune Photosensitivity. Front Immunol 9, 2430.

54. Ongusaha, P.P. et al. (2008) Identification of ROCK1 as an upstream activator of the JIP-3 to JNK signaling axis in response to UVB damage. Sci Signal 1 (47), ra14.

55. Reefman, E. et al. (2006) Is disturbed clearance of apoptotic keratinocytes responsible for UVB-induced inflammatory skin lesions in systemic lupus erythematosus? Arthritis Res Ther 8 (6), R156.

56. Colonna, L. et al. (2014) Beyond apoptosis in lupus. Curr Opin Rheumatol 26 (5), 459-66.

57. Sim, J.H. et al. (2021) Immune Cell-Stromal Circuitry in Lupus Photosensitivity. J Immunol 206 (2), 302-309.

58. Marathe, G.K. et al. (2005) Ultraviolet B radiation generates platelet-activating factor-like phospholipids underlying cutaneous damage. J Biol Chem 280 (42), 35448-57.

59. Zawrotniak, M. et al. (2019) UVA and UVB radiation induce the formation of neutrophil extracellular traps by human polymorphonuclear cells. J Photochem Photobiol B 196, 111511.

60. Yipp, B.G. and Kubes, P. (2013) NETosis: how vital is it? Blood 122 (16), 2784-94.

61. Papayannopoulos, V. (2018) Neutrophil extracellular traps in immunity and disease. Nat Rev Immunol 18 (2). 
62. Boeltz, S. et al. (2019) To NET or not to NET:current opinions and state of the science regarding the formation of neutrophil extracellular traps. Cell Death Differ 26 (3), 395-408.

63. Fuchs, T.A. et al. (2007) Novel cell death program leads to neutrophil extracellular traps. J Cell Biol 176 (2), 231-41.

64. Halverson, T.W. et al. (2015) DNA is an antimicrobial component of neutrophil extracellular traps. PLoS Pathog 11 (1), e1004593.

65. Yang, H. et al. (2016) New Insights into Neutrophil Extracellular Traps: Mechanisms of Formation and Role in Inflammation. Front Immunol 7, 302.

66. Neubert, E. et al. (2020) The power from within - understanding the driving forces of neutrophil extracellular trap formation. J Cell Sci 133 (5).

67. Thiam, H.R. et al. (2020) Cellular Mechanisms of NETosis. Annu Rev Cell Dev Biol 36, 191218.

68. Wesolowska, N. et al. (2020) Actin assembly ruptures the nuclear envelope by prying the lamina away from nuclear pores and nuclear membranes in starfish oocytes. Elife 9.

69. Chugh, P. and Paluch, E.K. (2018) The actin cortex at a glance. J Cell Sci 131 (14).

70. Sharma, M.R. et al. (2020) Effect of TNFalpha blockade on UVB-induced inflammatory cell migration and collagen loss in mice. J Photochem Photobiol B 213, 112072.

71. Yang, D. et al. (2000) LL-37, the neutrophil granule- and epithelial cell-derived cathelicidin, utilizes formyl peptide receptor-like 1 (FPRL1) as a receptor to chemoattract human peripheral blood neutrophils, monocytes, and T cells. J Exp Med 192 (7), 1069-74.

72. Kurosaka, K. et al. (2005) Mouse cathelin-related antimicrobial peptide chemoattracts leukocytes using formyl peptide receptor-like 1/mouse formyl peptide receptor-like 2 as the receptor and acts as an immune adjuvant. J Immunol 174 (10), 6257-65.

73. Skopelja-Gardner, S. et al. (2020) The early local and systemic Type I interferon responses to ultraviolet B light exposure are cGAS dependent. Sci Rep 10 (1), 7908.

74. Seery, J.P. (2000) IFN-gamma transgenic mice: clues to the pathogenesis of systemic lupus erythematosus? Arthritis Res 2 (6), 437-440.

75. Sarkar, M.K. et al. (2018) Photosensitivity and type I IFN responses in cutaneous lupus are driven by epidermal-derived interferon kappa. Ann Rheum Dis.

76. Oke, V. et al. (2019) High levels of circulating interferons type I, type II and type III associate with distinct clinical features of active systemic lupus erythematosus. Arthritis Res Ther 21 (1), 107.

77. Isgro, J. et al. (2013) Enhanced rho-associated protein kinase activation in patients with systemic lupus erythematosus. Arthritis Rheum 65 (6), 1592-602.

78. Rozo, C. et al. (2016) Targeting the RhoA-ROCK pathway to reverse T-cell dysfunction in SLE. Ann Rheum Dis 76 (4), 740-747. 


\section{Figure Legends}

Figure 1. Disruption of actin cytoskeleton or actomyosin cytoskeletal networks attenuated NETosis in neutrophils. A,B. dPMNs (A) or primary human neutrophils from different healthy donors (B) were exposed to $50 \mathrm{nM}$ PMA for $3 \mathrm{~h}$ without or with $30 \mathrm{~min}$ pretreatment by $10 \mu \mathrm{M}$ Cytochalasin D (Cyto D), $50 \mu \mathrm{M}$ Blebbistatin, or $1 \mu \mathrm{M}$ ML7, then detection of NETosis with Sytox Green staining by microplate reader. C. Primary bone marrow (BM) mouse neutrophils from different C57BL/6 wild type (WT) mice were treated with $10 \mu \mathrm{M}$ PAF (a physiological stimulus) for $3 \mathrm{~h}$, without or with 30 min pretreatment by $10 \mu \mathrm{M}$ Cyto D, $50 \mu \mathrm{M}$ Blebbistatin, or $1 \mu \mathrm{M}$ ML7, and stained by cell permeable SYTO Red and cell impermeable Sytox Green, then detected by fluorescent microscopy $(\mathbf{C})$. Images were taken by confocal microscopy, followed by automated quantification of NETs in 5-6 non-overlapping areas per well using ImageJ for calculation of \% cells with NET formation. D. Representative images for the effects of Cyto D, Blebbistatin, and ML7 on nuclear translocation of PKC $\alpha$ and nuclear lamina (lamin B) disintegration in dPMNs exposed to $50 \mathrm{nM}$ PMA for $1 \mathrm{~h}$ without or with pretreatment by above inhibitors, then stained for lamin $\mathbf{B}$ and PKCa with corresponding antibodies. Scale bars, $10 \mu \mathrm{m}$. Panel $\mathbf{E}$ displays the schematic illustration of the involvement of actin cytoskeleton and actomyosin cytoskeletal networks in NETosis and the corresponding inhibitors. All results are representative of 3 or more biological replicates. Panels A-C display means \pm SEM, $\quad \mathrm{P}<0.01$ by ANOVA; displayed are significance levels for individual pairwise comparisons by SNK test.

Figure 2. PKCa nuclear translocation and actin cytoskeleton assembly were regulated by Rho kinase 1 (ROCK1). A. Representative images for the time course study of PKCa nuclear 
translocation, subsequent lamin B disassembly and nuclear lamina disintegration, and NET release in dPMNs exposed to $50 \mathrm{nM}$ PMA for 0-180 min, then stained simultaneously for DNA, lamin B (probed by primary anti-lamin B, and FITC-labeled secondary antibody), and PKC $\alpha$ (primary antiPKC $\alpha$, and PE-labeled secondary antibody) under confocal fluorescent microscopy. Scale bars, 10 $\mu \mathrm{m}$. B. Representative flow cytometric histogram for the time course of F-actin polymerization in dPMNs exposed to $50 \mathrm{nM}$ PMA for 0-180 min, the pemeabilized cells were probed with phalloidin-RFP. Panel $\mathbf{C}$ shows the time course of ROCK activation in dPMNs exposed to $50 \mathrm{nM}$ PMA for 0-180 min, detected by ROCK activity ELISA. Panel D indicates F-actin polymerization detected by RFP-labeled phalloidin with flow cytometry in dPMNs exposed to 50 nM PMA for 60 min without or with pretreatment by $50 \mu \mathrm{M}$ ROCK inhibitors (Y27632, HA1077, AS1892802) for 30 min. E. Representative images for the effect of ROCK inhibition on PKC $\alpha$ nuclear translocation and nuclear envelope disintegration in dPMNs exposed to PMA for 60 min without or with pretreatment by ROCK inhibitors, then stained for lamin B and PKC $\alpha$ with their corresponding Abs. Scale bar, $10 \mu \mathrm{m}$. Light blue arrows in panels $\mathbf{A}, \mathbf{E}$ indicate the disintegration/rupture of nuclear lamina at 60 min after PMA treatment, while white arrows (A) indicate the rupture site of the nuclear envelope where the decondensed chromatin released for NET formation in later time points. Panel $\mathbf{F}$ displays the schematic illustration of the involvement of actin cytoskeleton, actomyosin cytoskeletal networks, their upstream signaling pathways, and ROCK, as well as their corresponding inhibitors in NETosis. All results are representative of 3 or more biological replicates, and panels $\mathrm{A}, \mathrm{B}, \mathrm{D}, \mathrm{E}$ are the representative of the replicates. In panel $\mathrm{C}, \mathrm{P}^{*} *<0.01$, $\mathrm{P}^{* * *}<0.001$ vs $0 \mathrm{~min}, \mathrm{P}^{\dagger \dagger}<0.01$ vs $60 \mathrm{~min}$. 
Figure 3. Inhibition of ROCK1 attenuated NETosis in neutrophils. Panels A,B show the results of NETosis of either dPMNs (A) or primary human neutrophils from different healthy donors (B), which were exposed to $50 \mathrm{nM}$ PMA for $3 \mathrm{~h}$ without or with $30 \mathrm{~min}$ pretreatment by $50 \mu \mathrm{M}$ ROCK inhibitors (Y27632, HA1077, AS1892802), then detection of NET formation by microplate reader. C-E, Summary analysis (C,D) and (E) representative of NET formation of mouse BM neutrophils from different WT mice with pretreatment by different ROCK inhibitors $(\mathbf{C})$, or BM neutrophils from WT vs ROCK $1^{+/-}$mice $(\mathbf{D}, \mathbf{E})$, and stimulated without or with $10 \mu \mathrm{M}$ PAF for $3 \mathrm{~h}(\mathbf{C}-\mathbf{E})$, and stained with SYTO Red and Sytox Green. Images were taken by confocal microscopy, and \% cells with NET formation was analyzed as described in Fig 1. All results are representative of 3 or more biological replicates, and panel E is representative images of the replicates. Scale bars, $100 \mu \mathrm{m}$. Panels A,B,C,D display mean \pm SEM. In these panels, $\mathrm{P}<0.01$ by ANOVA; displayed are significance levels for individual pairwise comparisons by the SNK tests.

Figure 4. Hematopoietic specific ROCK1 deficiency protected mice from UVB-induced skin inflammation in BMT-ROCK1 $1^{+/}$mice. A-E. Representative H\&E staining images (A) and summary analysis of the thickness of epidermis and dermis $(B, C)$, infiltration of inflammatory (nucleated) cells in the dermis and subcutis (D) and their correlation with dermis thickness (E) based on H\&E staining of skin sections of BMT-WT vs BMT-ROCK1 ${ }^{+/-}$mice that were irradiated without (sham) or with UVB. F-H. Representative images (F) and summary analysis of NETosis $(\mathrm{G})$, and its correlation with skin thickness $(\mathrm{H})$ in skin of BMT-WT vs BMT-ROCK1 ${ }^{+/-}$mice that were irradiated without (sham) or with UVB. I. Neutrophil infiltration per unit area (I) in skin of UVB-irradiated BMT-WT vs BMT-ROCK1 ${ }^{+-}$mice. J. Relative migration analysis of BM neutrophils of WT vs ROCK1 $1^{+/-}$mice was conducted with Boyden chamber by stimulation with 
CXCL-1. K. Percentage of NETotic cells among neutrophils in skin of UVB-irradiated BMT-WT vs BMT-ROCK1 ${ }^{+/-}$mice. For panels F-I,K, skin sections were stained by antibodies against Ly6G (PE) neutrophil marker and citrullinated histone H3 (citH3, FITC), and DAPI for DNA. In panels A,F, Scale bars, $100 \mu \mathrm{m}$. All results are representative of 4-8 biological replicates. In the panels $\mathrm{B}, \mathrm{C}, \mathrm{D}, \mathrm{G}, \mathrm{P}<0.01$ by $\mathrm{ANOVA}$; displayed are significance levels for individual pairwise comparisons by SNK test. Panels I,J,K were analyzed by student's t-test.

Figure 5. Hematopoietic specific ROCK1 deficiency attenuated skin inflammation with decreased exhibition of NET-associated pro-inflammatory cytokines in UVB-irradiated BMT-ROCK1 ${ }^{+/}$ mice. A-I. Summary analyses $(\mathbf{A}, \mathbf{D}, \mathbf{F}, \mathbf{H})$ and representative images $(\mathbf{B}, \mathbf{C}, \mathbf{E}, \mathbf{G}, \mathbf{I})$ of skin sections of BMT-WT vs BMT-ROCK1+/- mice that were irradiated without (sham) or with UVB, and stained by PE-conjugated rat anti-mouse Ly6G Ab, and FITC labeled rat anti-mouse IL-17A, TNF $\boldsymbol{\alpha}$, IFN $\gamma$, or IFNa. DNA was stained by DAPI for panels A-I. Yellow arrows indicate NETotic neutrophils, and white arrows show non-netting neutrophils in panels B,C,E,G,I. Scale bars, $100 \mu \mathrm{m}$. All results are representative of 4-7 biological replicates. In the panels A,D,F,H, $\mathrm{P}<0.01$ by ANOVA; displayed are significance levels for individual pairwise comparisons by the SNK test.

Figure 6. Genetic deficiency of ROCK1 decreased formation of NETs and attenuated their display of pro-inflammatory cytokines in neutrophils from ROCK1 deficient mice. A-H. Summary analyses $(\mathbf{A}, \mathbf{C}, \mathbf{E}, \mathbf{G})$ and representative images $(\mathbf{B}, \mathbf{D}, \mathbf{F}, \mathbf{H})$ of BM neutrophils from ROCK ${ }^{+/-}$mice or their WT littermates were treated without or with $10 \mu \mathrm{M}$ PAF (for IFN $\alpha$ induction in G-H, treatment with PAF and isolated NETs from cultured neutrophils of MRL/lpr mice) for 20h, 
following by fixation and immunocytochemistry staining of neutrophils by PE-conjugated antimouse Ly6G, FITC labeled anti-mouse IL-17A, TNF $\alpha$, IFN $\gamma$, or IFN $\alpha$. DNA was stained by DAPI. Scale bars, $50 \mu \mathrm{m}$. Arrows indicate NETs with extracellular display of different cytokines.

All results are representative of 5-7 biological replicates. In the panels $A, C, E, G, P<0.01$ by ANOVA; displayed are significance levels for individual pairwise comparisons by the SNK test. 
Figure 1.
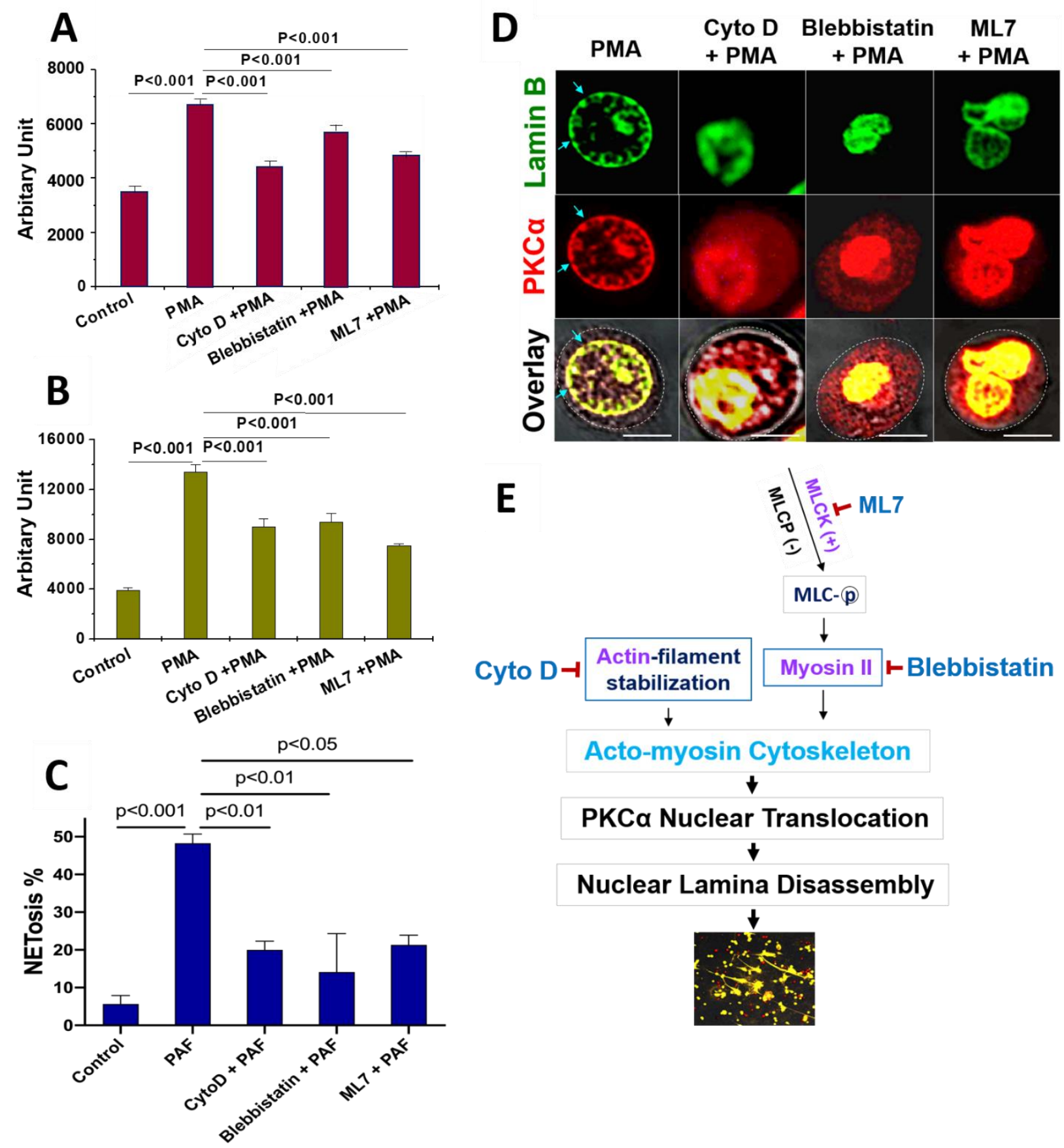
Figure 2.
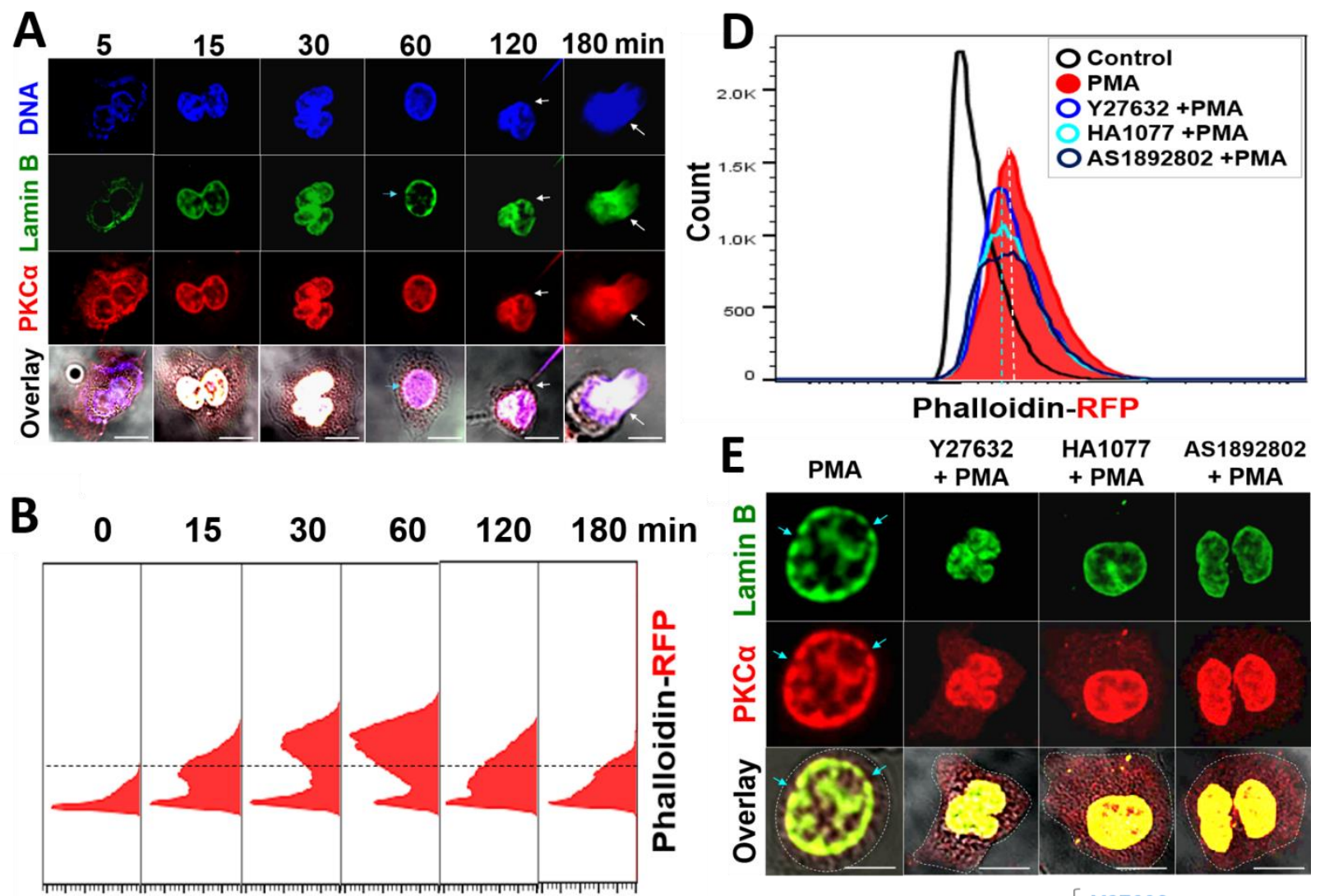

\section{Count}
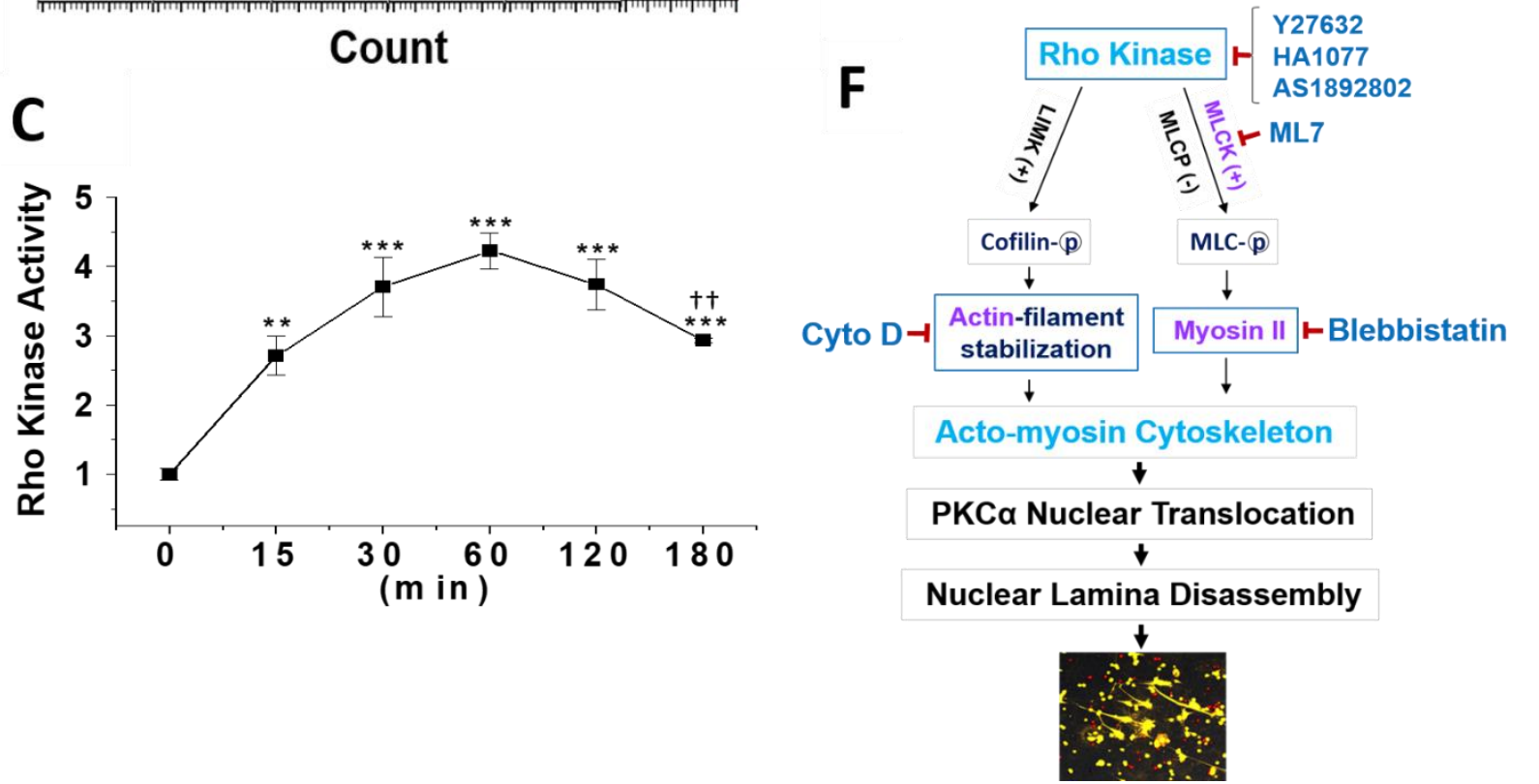
Figure 3.
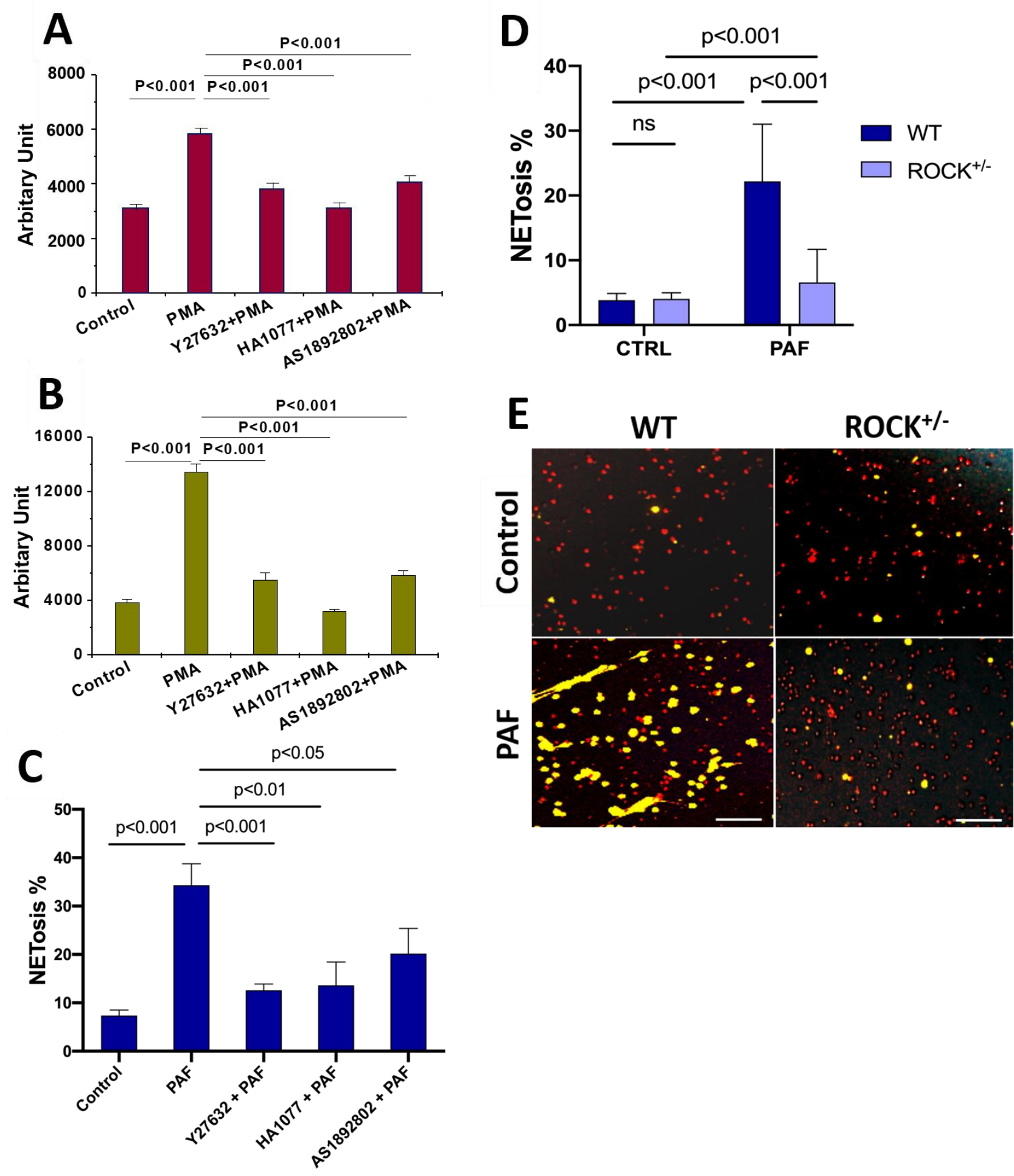


\section{Figure 4.}
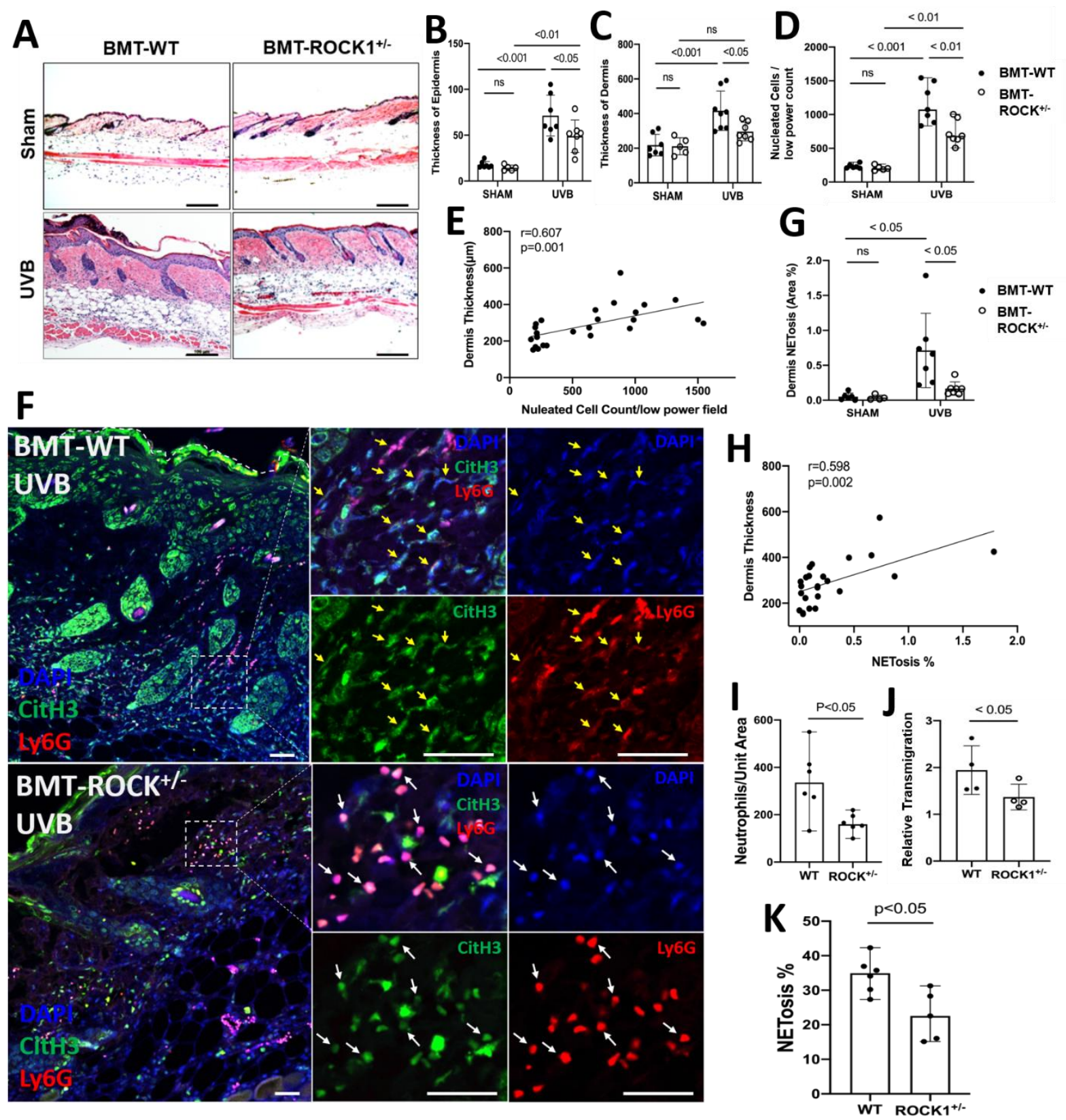


\section{Figure 5.}
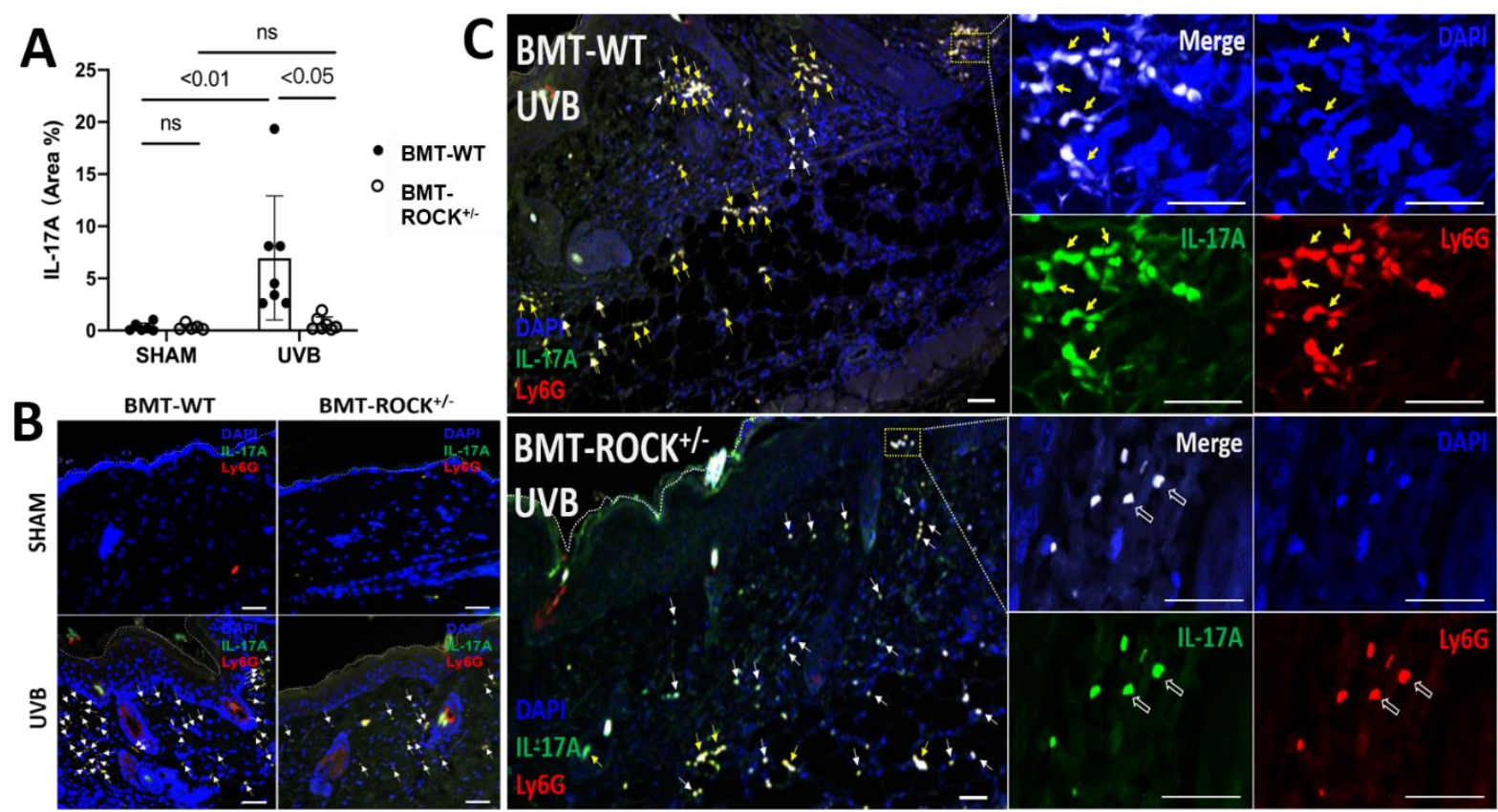

D
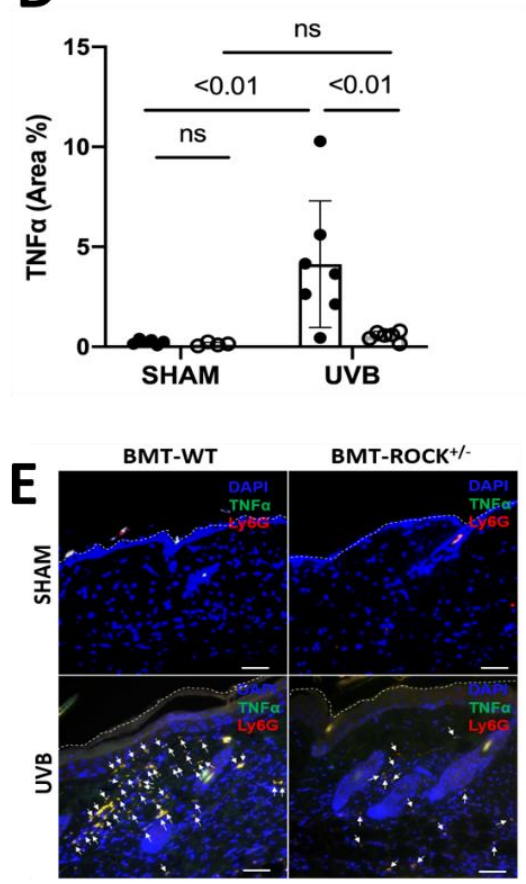

$\mathbf{F}$

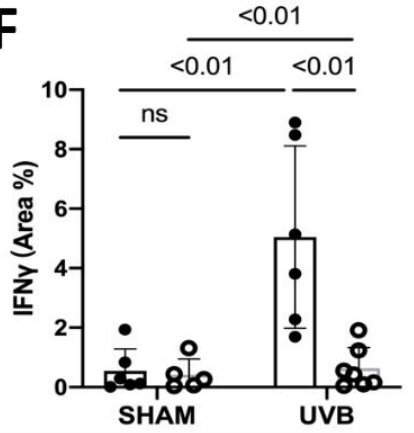

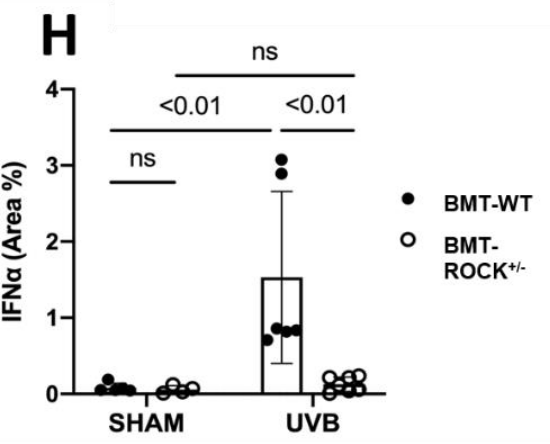
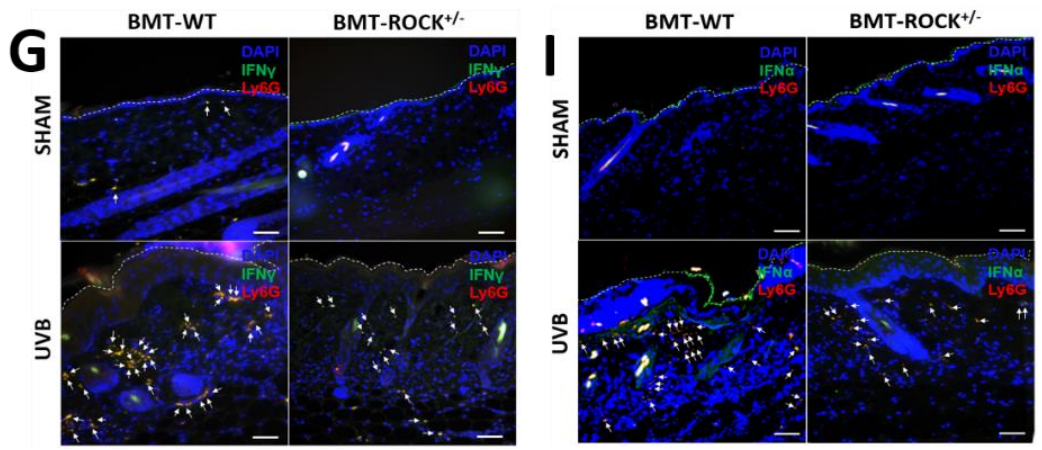
Fig 6.
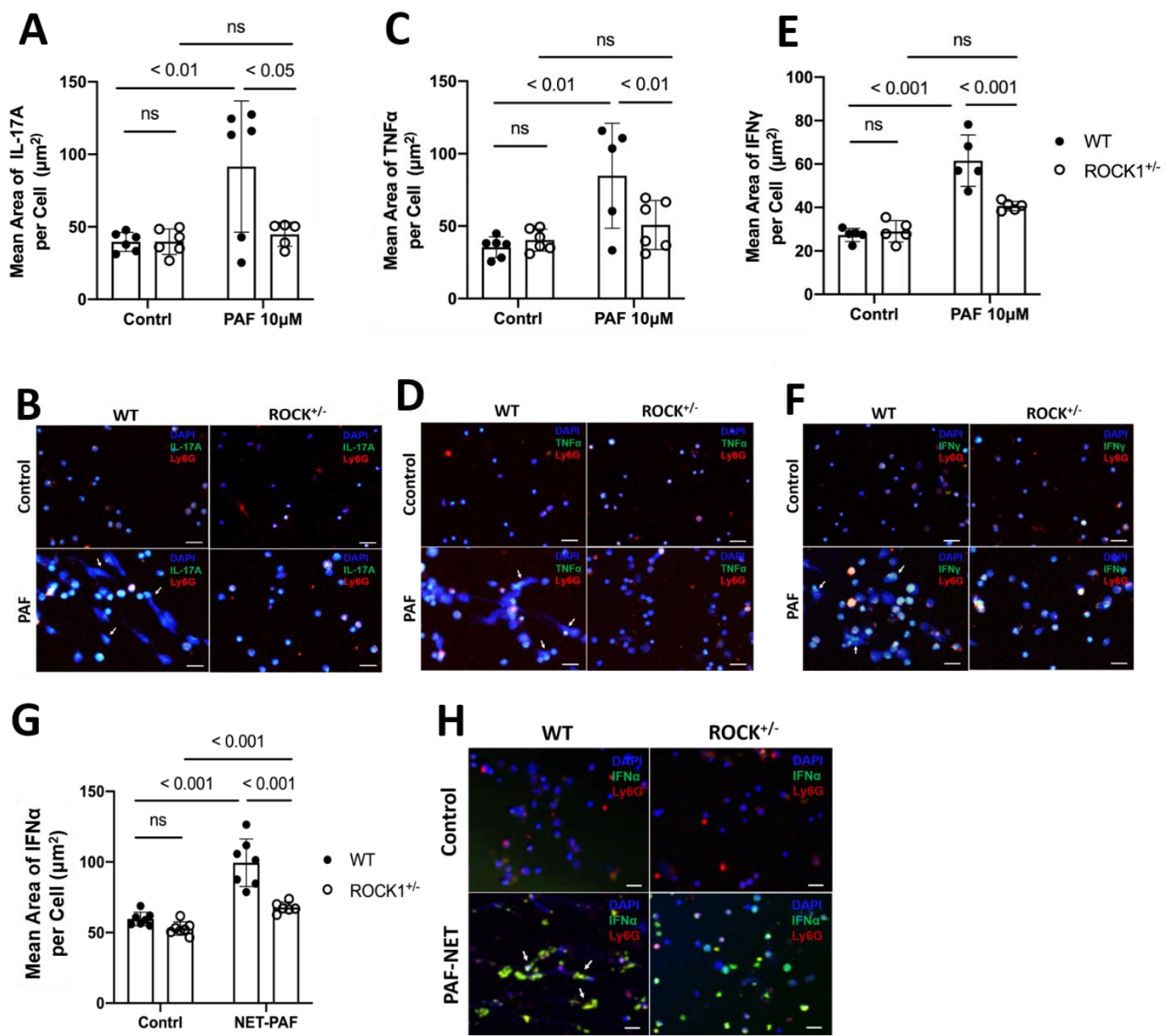


\section{Supplemental Materials}

Rho Kinase regulates neutrophil NET formation that is involved in UVB-induced skin inflammation

Running title: Rho kinase and NETosis

\section{Supplemental Methods}




\section{Supplemental Figures}
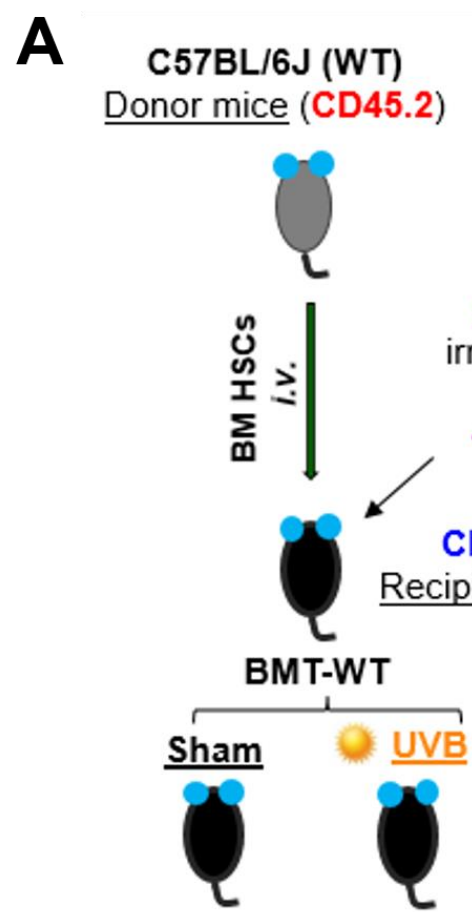

ROCK1 $^{+1-}$

Donor mice (CD45.2)

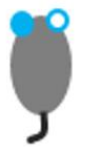

y-rav

irradiation

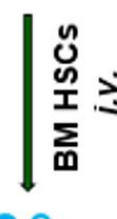

CD45.1

Recipient mice
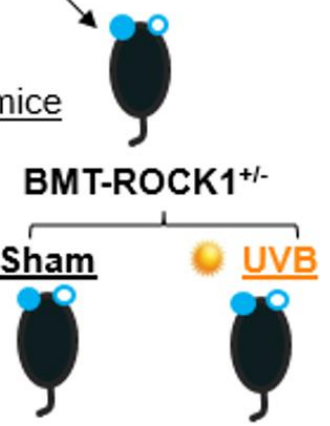

B

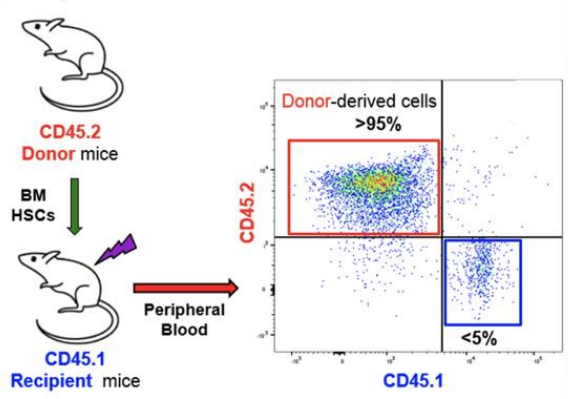

C

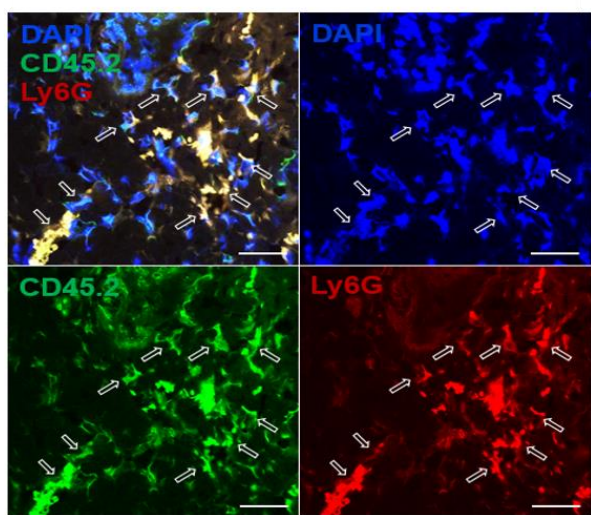

Supplmental Fig 1. Generation of mice with hematopoietic specific deficiency of ROCK1. Panel A displays the experimental design of the BMT-WT and BMT-ROCK $1^{+-}$mice that were irradiated without or with UVB. Panel $\mathbf{B}$ is a representative dot plots of flow cytometry analysis for all BMT experimental mice for detection of reconstitution rate of donor-derived cells (CD45.2) in peripheral blood of recipient mice (CD45.1). C. The representative image of immunohistochemistry staining of skin lesion from UVB-irradiated CD45.1 recipient mice with BMT of HSCs from WT donors, stained by antibodies against Ly6G (PE), CD45.2 (FITC) or DAPI. Arrows indicate the reconstituted neutrophils that were infiltrated to the inflamed skin of recipient mice with UVB irradiation. 

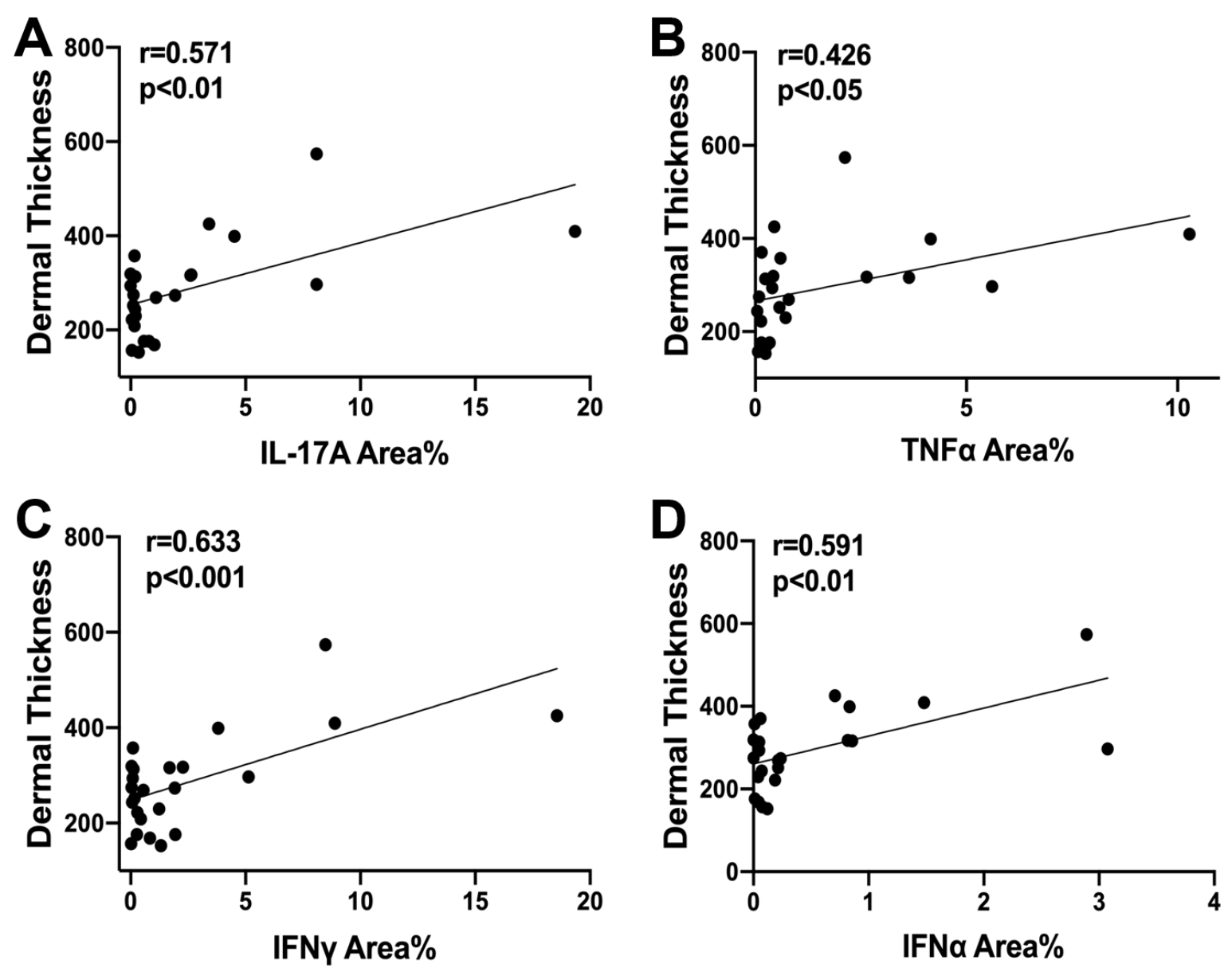

Supplmental Fig 2. Correlation between NET-associated IL-17A (A), TNF $\alpha$ (B), IFN $\gamma$ (C), or IFN $\alpha$ (D) with dermal thickness in all groups of BMT experimental mice. Pearson correlation analysis was conducted to analyze association between NET-associated cytokines and dermal skin thickness. 